\title{
Thin film dynamics: theory and applications
}

\author{
Andrea L. Bertozzi and Mark Bowen \\ Department of Mathematics \\ Duke University \\ Durham, NC 27708 USA
}

February 13, 2002

\begin{abstract}
This paper is based on a series of four lectures, by the first author, on thin films and moving contact lines. Section one presents an overview of the moving contact line problem and introduces the lubrication approximation. Section two summarizes results for positivity preserving schemes. Section three discusses the problem of films driven by thermal gradients with an opposing gravitational force. Such systems yield complex dynamics featuring undercompressive shocks. We conclude in section four with a discussion of of dewetting films.
\end{abstract}

\section{Thin films and moving contact lines}

1 The motion of a liquid under the influence of surface tension is a phenomenon we experience every day when we take a shower, drink a cup of coffee, or turn on the windshield wipers. All of these real world situations involve not only the motion of the liquid and the surrounding air, but also their interaction with adjacent hard surfaces. As we know from waxing our cars and cooking with non-stick cookware, the dynamics of a fluid coating a solid surface depend heavily on the surface chemistry. Many industrial processes, ranging from spin coating of microchips to de-icing of airplane wings, rely on the ability to control these interactions.

An ongoing challenge is to explain the underlying physics of the motion of a contact line (see Figure 1), a triple juncture of the air/liquid, liquid/solid, and air/solid interfaces. When the system is at rest, the three interfacial energies, determined by the energy per unit area $\gamma_{S}(S=a l, l s$, or $a s)$, are in balance and an equilibrium contact angle $\theta$ satisfies Young's law $[37]$

$$
\gamma_{a s}-\gamma_{l s}-\gamma_{a l} \cos \theta=0 .
$$

However, the dynamically evolving contact line requires much more subtle modeling involving the interaction between multiple length scales due to the underlying fluid dynamics.

The classical theory of fluids [2] tells us that when a viscous fluid meets a solid boundary, the correct model for the boundary is a 'no-slip' condition on the liquid/solid interface ${ }^{2}$.

\footnotetext{
${ }^{1}$ Parts of this section are excerpted from the review article [26].

${ }^{2}$ see [50] pp. 676-680 for a comprehensive discussion of the history of the 'no-slip' boundary condition
} 


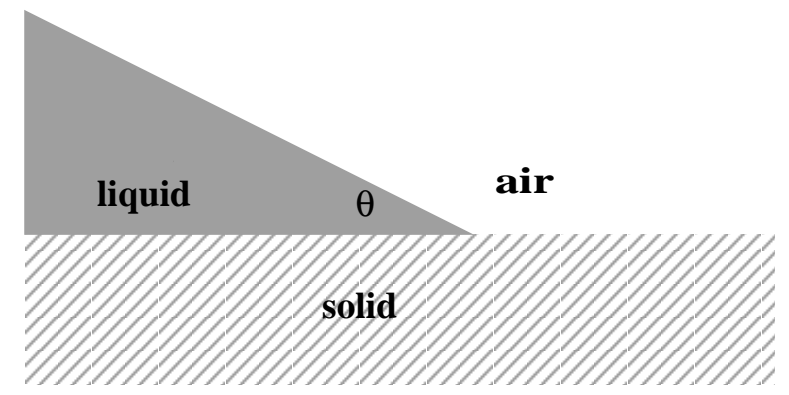

Figure 1: Simple trigonometry is all that is needed to derive Young's law (1) -the equation satisfied by the equilibrium contact angle. However, in cases like complete wetting or driven contact lines, a dynamic model is required. Physicists have been fascinated by this problem for decades.

Mathematically, this requires setting the fluid velocity to be zero on the solid boundary. While this makes sense for the Navier-Stokes equations (describing the motion of an incompressible fluid) in bounded domains, its relevance to the moving contact line problem is dubious. The difficulty is that a moving contact line coupled with a no-slip condition on the liquid solid interface results in a multi-valued velocity field at the contact line. The velocity field is not well defined because the limit along the solid surface is zero, while the limit along the air/liquid interface (which is moving) is non-zero. Regardless of the liquid/air interface model, the tangential component of force exerted by the fluid on the solid diverges whenever the velocity is multi-valued at the contact line $[59,39]$. The fact that such a paradox exists is hardly surprising. We should not expect to find a self-consistent universal hydrodynamic model that does not incorporate the surface chemistry.

Several models have been proposed to study the motion of moving contact lines. All of them involve adding an additional effect on a microscopic length-scale. The two that we discuss in the next paragraphs are (1) weakening the no-slip boundary condition via a slip condition effective at small scales and (2) incorporating the effect of long range van der Waals forces (described below) between the liquid and solid. Perhaps the simplest context in which to test these theories is that of thin viscous coating flows. For such problems, a lubrication approximation simplifies bulk flow fluid dynamics, as given by the Navier-Stokes equations, to a single equation, relating the depth averaged horizontal fluid velocity to the shape of the air/liquid interface.

The Navier slip condition ${ }^{3}$ near the liquid solid interface demands that the velocity at the interface be proportional to its normal derivative:

$$
v=\frac{\partial v}{\partial z} k(h), \quad z=0 .
$$

Here $v$ is the horizontal fluid velocity and $z$ is the variable in the direction normal to the solid surface. The parameter $k(h)$ is a slip parameter and can depend on the thickness $h$ of the film. We can think of condition (2) as a generalization of the standard "no-slip" condition which corresponds to $k \equiv 0$ in $(2)$.

\footnotetext{
${ }^{3}$ a generalization of the "no-slip" condition in which the fluid is allowed to slip tangentially along the solid boundary
} 
Another choice for removing the singularity due to no-slip is to include microscopic scale forces, in the form of long range van der Waals (VW) interactions between the liquid and solid surfaces, near the contact line. The most systematic way to do this is via an additional body force in the fluid of the form (see the review articles [37] sec. II. D. and [79] sec. II. E. )

$$
\Pi(h)=A_{D} h^{D-5},
$$

where $D$ is the "dimension" of the substrate ${ }^{4}$. Here $A_{D}$ is the Hamaker constant, which depends on the dimension and also on the strength of the interaction between the liquid and solid particles. The sign of $A_{D}$ depends on whether the VW forces are attractive or repulsive. The particular power in (3) comes from assuming that the particles in the fluid interact with particles in the solid substrate via a "non-retarded" potential. See the references listed above and the references therein for more discussion of this and other potentials.

\subsection{The basic equation}

In its simplest form, the lubrication approximation can be derived from a systematic rescaling and asymptotic expansion of the Navier-Stokes equations in the limit of vanishing capillary number $C_{a}$ and Reynold's number $R_{e}$, two dimensionless parameters given by

$$
C_{a}=\frac{3 \nu V}{\gamma}, \quad R_{e}=\frac{\rho V h_{0}}{\nu}
$$

where $\nu$ is viscosity, $\gamma$ is surface tension, $\rho$ is the density of fluid, $h_{0}$ a characteristic height of the film, and $V$ is a characteristic velocity of the film. In this case, when the slip model (2) is used, the nondimensional lubrication equation

$$
\begin{aligned}
h_{t}+\nabla \cdot(f(h) \nabla \Delta h) & =0, \\
f(h) & =h^{3}+b^{3-p} h^{p}
\end{aligned}
$$

results. Here $b$ represents a dimensionless slip length. Greenspan [52] derives (5) with $b>0$, "singular slip" $p=1>0$ and $\Pi(h)=0$. Haley and Miksis [57] consider this same model with different integer values of $p=0,1,2$. The VW model (3) results in a similar equation with a second order term that includes the effect of $\Pi(h)$.

The remainder of this section discusses basic mathematical properties of equations of the form (5). Section 2 discusses numerical schemes for this problem. Section 3 considers the problem when convective terms are included. Section 4 addresses the dewetting problem and pattern formation, in which effects like (3) are included.

The engineering and applied mathematics literature has had much discussion of the most appropriate boundary conditions for an equation of the form (5) near the vicinity of a contact line, especially in the context of a slip model (see [79], sections II B and V and references therein. See also Hocking J. Fl. Mech. v. 239, 1992). During the past eight years, mathematicians working in the field of nonlinear PDEs have also become interested in this problem from a more analytical point of view.

Equation (5) is a fourth order degenerate diffusion equation. Diffusion equations arise in models of physical problems in which some quantity spreads or smears out. The classical

\footnotetext{
${ }^{4}$ i.e. $D=2$ for a film on a two dimensional surface and $D=1$ for a flow in the plane, bounded by a line
} 
example is the heat equation $u_{t}=\nabla \cdot k \nabla u$ where the diffusion coefficient $k$ controls the rate at which heat "diffuses" through the medium. In order to understand the contact line models proposed for equation (5) we need to understand something about degenerate diffusion equations, where $k$ depends on the solution $h$, so that $h \rightarrow 0$ as $k \rightarrow 0$. A well-known family of second order degenerate diffusion equations are the porous media equations [80]:

$$
h_{t}-\Delta(\Phi(h))=0
$$

where $\Phi^{\prime}(h)>0$ for $h>0$ and $\Phi(h) \sim h^{m}$ as $h \rightarrow 0$. Here, $m>1$ makes the equation degenerate.

The Laplacian operator $\Delta$ endows the evolution equation (6) with some important properties:

1. Instantaneous smoothing of the solution in regions of positive $h$, since the equation is uniformly parabolic (i.e. the "diffusion coefficient" is bounded away from zero) where the solution is bounded away from zero.

2. Maximum principle. The solution is bounded from above and below by its initial data. This is true regardless of whether or not $m=1$ (linear heat equation) or $m>1$.

3. Well-posed distribution solutions for smooth nonnegative initial data.

4. Finite speed of propagation of the support of the solution.

The last property is special to degenerate diffusion $(m>1)$. The heat equation $(m=1)$ has infinite speed of propagation of the support.

Consider now a fourth order analogue of (6) that arises in the thin film problem (as in (5))

$$
h_{t}+\nabla \cdot(f(h) \nabla \Delta h)=0 .
$$

In the context of thin film dynamics, we are interested in questions of existence, uniqueness, and finite speed of propagation of the support of weak solutions of equations of the form (7). The important difference from the case (6) is that (7) is fourth order as opposed to second order. It is diffusive so we have property (1), instantaneous smoothing where the solution is positive, but property (2), the maximum principle, is far from guaranteed. Indeed, if we take the nondegenerate case $f(h)=1$, then solutions can change sign. This can be seen by simply examining the heat kernel for fourth order diffusion in $R^{D}$ and noting that unlike the wellknown second order heat kernel, the fourth order heat kernel has an oscillatory exponentially decaying tail that changes sign. It is not immediately apparent why the nonlinear problem (7) should have solutions that preserve their sign.

What is remarkable is that the nonlinear structure of (7) endows it with a positivity preserving property. In particular, one can show for one space dimension $[13,27]$ with $f(h)=$ $h^{n}$ with $n \geq 3.5$ on a periodic spatial domain, if the initial condition $h_{0}$ is positive, then the solution $h(x, t)$ is guaranteed to stay positive. In fact, one can derive an a priori pointwise lower bound depending only on the $H^{1}$ norm and minimum value of the initial data. This form of a weak maximum principle is due to the nonlinear structure of (7), not the structure of the fourth order diffusion operator. It is noteworthy that other fourth order degenerate diffusion equations with different nonlinear structure have weak solutions that change sign 
[12]. Despite the positivity preserving property for large $n$, solutions of (7) with smaller values of $n$ computationally exhibit finite time singularities of the form $h \rightarrow 0$. We discuss this phenomenon in more detail in the next section. The fact that such singularities may occur makes the development of a weak solution theory, analogous to that of the porous media equation, all the more difficult. Over the past few years, mathematical machinery, largely involving energy estimates and nonlinear entropies, has been developed to address these problems. Such methods have been used to prove weak maximum principles, derive existence results for weak (nonnegative) solutions, and to prove results concerning the finite speed of propagation of the support of nonnegative solutions $[15,16]$.

\subsection{Finite time singularities and similarity solutions of lubrication equa- tions}

The study of finite time singularities and similarity solutions of (7) began in the early 1990's by a group at the University of Chicago. The original project addressed the breakup of a thin neck in the Hele Shaw cell when forced by an external pressure. The experiment, performed by Goldstein at Princeton [36], can be modeled, via lubrication theory, by a fourth order degenerate diffusion equation in one space dimension:

$$
h_{t}+\left(h^{n} h_{x x x}\right)_{x}=0
$$

with "pressure" boundary conditions $h( \pm 1)=1, h_{x x}( \pm 1)=-p$, and $n=1$. Here $h$ denotes the thickness of the fluid neck, in the direction parallel to the plates (resulting from a $2 \mathrm{D}$ model). Carefully resolved numerical computations revealed that this problem yields both finite and infinite time singularities of the form $h \rightarrow 0$, for various initial data [38].

What is unusual about the structure of these singularities is that, while they locally have self-similar structure of the form

$$
h(x, t) \sim \tau(t) H\left(\frac{x-a(t)}{l(t)}\right),
$$

the time dependences $\tau(t), l(t)$, and $a(t)$ are not completely determined by dimensional analysis of the PDE. Such anomalous 'second type' scaling [7] arises in problems such as the focusing solution of the porous media equation [4] where one solves a nonlinear eigenvalue problem to determine the profile $H$ and the relationship between scales $\tau(t)$ and $l(t)$. However, in the case of the lubrication singularities, the scaling relations are typically found by asymptotic matching involving the boundary conditions or matching to an intermediate length-scale [27].

The original work on the Hele-Shaw problem was subsequently generalized to the case of variable nonlinearity (other values of $n$ ) with other boundary conditions $[27,3]$ and also to different fourth order degenerate PDEs [25]. The similarity solutions observed were found using careful numerical computations involving adaptive mesh refinement near the singularity. Scaling was then observed for many decades in $h_{m i n}$, the minimum value of the solution. All of these papers make the following observations:

- All observed finite time singularities of the form $h \rightarrow 0$ as $t \rightarrow t_{c}$ involve second type scaling behavior in which the similarity solution described in (9) satisfies the quasi-static equation $\left(f(h) h_{x x x}\right)_{x}=0$. 
- Several kinds of similarity solutions can occur for different initial conditions for the same equation. The far field dynamics of the structure may cause the similarity solution to destabilize, at arbitrarily small length-scales, from one type of similarity solution to another [3].

As an example, consider the equation (8) with $n=1 / 2$, periodic boundary conditions on $[-1,1]$, and initial condition

$$
h_{0}(x)=0.8-\cos (\pi x)+0.25 \cos (2 \pi x) .
$$

The solution develops a finite time 'pinching' singularity in which $h \rightarrow 0$ with a simultaneous blow-up in the fourth derivative. The third derivative forms a step function (see Figure 2). Near the pinch-point, the solution has a leading order asymptotic form

$$
h(x, t) \approx c\left(t_{c}-t\right)+\frac{p\left(x-x_{c}\right)^{2}}{2},
$$

where $t_{c}$ is the time of pinch-off, $x_{c}$ is the pinch point, and the constant $p$ is the curvature of the interface at the time of and location of the singularity. The blow-up in higher derivatives can be seen only in terms of higher order than (11). The next term satisfies

$$
h_{x x x} \approx \frac{c x}{\sqrt{c\left(t_{c}-t\right)+p\left(x-x_{c}\right)^{2} / 2}}
$$

so that the local curvature $h_{x x}$ has the form

$$
p+\frac{c}{p} \sqrt{c\left(t_{c}-t\right)+p\left(x-x_{c}\right)^{2} / 2}
$$

This scaling structure of the singularity was confirmed via numerical simulations, using a self-similar adaptive mesh refinement code, that resolved the singularity over thirty orders of magnitude in $h\left(x_{c}, t\right)=h_{\min }$. The solution can be continued after the initial pinch-off singularity. We discuss the continuation of this particular example later in this paper (see Figures 3-5).

The fact that we can rigorously prove that finite time singularities cannot occur for $n \geq 3.5$ yet they are observed for certain $n<2$ suggests the existence of a critical exponent. This is a value $n_{*}>0$ for which solutions to (8) stay positive whenever $n>n_{*}$ and where finite time singularities are possible for $n \leq n_{*}$. To date, no such $n_{*}$ has been established, but numerical simulations suggest that $1<n_{*}<3.5$. It is also not clear if $n_{*}$ depends on boundary conditions. Since singularities appear to be localized, it is reasonable to expect that $n_{*}$ might be independent of boundary conditions. It is an interesting open question whether this behavior persists in higher space dimensions $(D>1)$.

\subsection{Entropies, positivity, and weak solutions}

In the previous section, we discussed the open problem of finding a 'critical exponent' associated with strictly positive initial data: equations with exponent larger than $n_{*}$ have solutions that remain strictly positive, while ones with exponents less than $n_{*}$ allow for the possibility of finite time singularities. In this section we introduce another critical exponent $n_{\text {crit }}$ which separates powers for which the solution has increasing support from powers for which the 


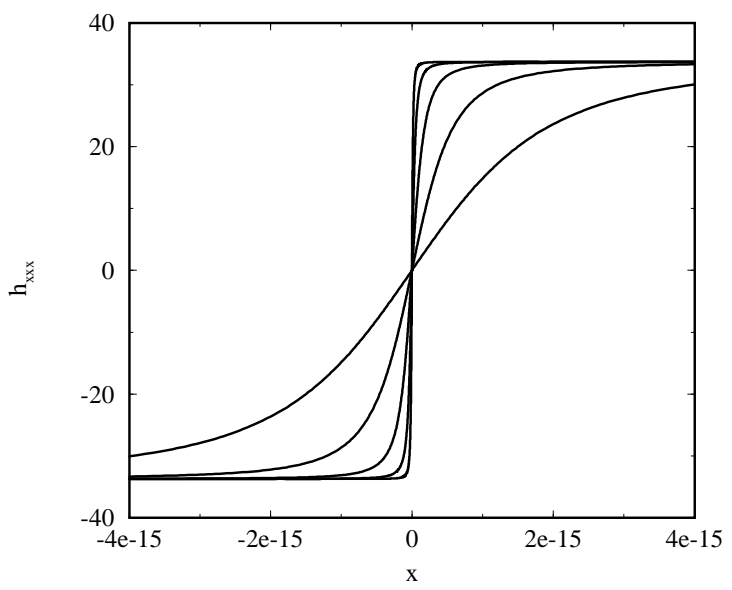

Figure 2: Onset of initial singularity in solution of (8) with $n=1 / 2$ and initial data (10). Formation of a jump discontinuity in the third derivative, shown as solid lines for successive times close to singularity. The solution can be continued after the initial pinch-off singularity.

support of the solution cannot increase. For the porous media equation (6), the support of the solution is always monotonically increasing, regardless of the size of $m>1$. For the 1D lubrication equation (8), the support of the solution cannot increase whenever $n \geq 4$ [11]. On the other hand, there are solutions with support that eventually increase to fill the entire domain for all $n<3$ [14]. It has been conjectured that the critical exponent for increasing support is $n_{\text {crit }}=3$, but this remains to be rigorously proved.

The exact value of the critical exponent has important ramifications for the moving contact line problem. If indeed $n_{\text {crit }}=3$ it tells us that there are no solutions of (5) with $b=0$ (no-slip) that describe a moving contact line. Such a result would not be surprising. It is consistent with the fact that such a scenario introduces a multi-valued velocity field that is known to produce infinities in the physics (hence the introduction of the slip terms that give $n<3)$.

Examples of known exact solutions support the conjecture that $n_{\text {crit }}=3$ in both one and two space dimensions. One class of examples are the 'source type solutions', solutions that start as a delta function at the origin and spread out in a self-similar way while conserving their mass. Starov [91] looked for such solutions to (7) with $f(h)=h^{3}$, in two space dimensions. He attempted to find a similarity solution to describe spreading drops under the influence of surface tension. What he discovered was that the resulting ODE for the similarity solution did not have any solutions of compact support. That is, there are no similarity solutions describing spreading drops with no-slip on the liquid/solid interface. Bernis and collaborators $[14,47]$ showed that in all space dimensions, source type solutions exist $\left(f(h)=h^{n}\right)$ for $n<3$ and they do not exist for $n \geq 3$. It is interesting that this critical exponent cannot be predicted from dimensional analysis of the equation; it is determined by properties of the ODE for the shape of the similarity solution. Traveling wave (i.e. advancing front) solutions [30] also change behavior at the critical exponent of $n=3$.

We now discuss some of the key ideas used to prove results about the PDE (7). A seminal 
paper in the mathematical development of the theory of weak solutions for lubrication-type equations is the work [13] by Bernis and Friedman. There they showed that, in addition to conservation of mass,

$$
\frac{\partial}{\partial t} \int h(x) d x=0
$$

and surface tension energy dissipation,

$$
\frac{\partial}{\partial t} \int|\nabla h|^{2}=-\int f(h)|\nabla \Delta h|^{2} d x
$$

equations of the type (7) possess a nonlinear entropy dissipation of the form

$$
\frac{\partial}{\partial t} \int G(h)=-\int|\Delta h|^{2} d x
$$

Here $G(h)$ is a convex function satisfying $G^{\prime \prime}(h)=1 / f(h)$. For the case $f(h)=h$ (e.g. HeleShaw) the entropy $\int G(h)$ is of the form $\int h \log h$; hence the name 'entropy' was born [24] to describe this object. Using the entropy (14), Bernis and Friedman proved that in one space dimension, the critical exponent $n_{*}$, above which singularity formation is forbidden, satisfies $n_{*}<4$. The proof uses the fact that (13) and conservation of mass imply an a priori bound on the $C^{1 / 2}$ norm of the solution while (14) insures a bound on $\int h^{2-n}$. This gives an a priori pointwise lower bound for the solution. It turns out that the integral $\int G(h) d x$ above is not the only dissipative entropy. In 1D we also have a family of entropies, satisfying

$$
\frac{\partial}{\partial t} \int G^{s}(h), \leq 0 \quad\left(G^{s}\right)^{\prime \prime}=\frac{h^{s}}{f(h)} .
$$

where $\frac{1}{2} \leq s \leq 1$. Taking $s \rightarrow \frac{1}{2}$ gives the upper bound $n_{*} \leq 3.5$ proved in [27].

The generalized entropies play an important role in the development of a weak existence theory for nonnegative solutions from nonnegative initial data, and for proving results on finite speed of propagation of the support. Uniqueness of weak solutions still remains an open problem. In the next section, we see how entropies have also been used recently to design numerical methods for solving these equations.

\section{Positivity preserving schemes}

The nonlinear structure of the PDE (7) presents a challenge in the design of efficient and accurate numerical methods. The degeneracy of (7) as $h \rightarrow 0$ can be the cause of a number of numerical instabilities. To compute a positive approximation of the solution, we may need computationally expensive local mesh refinement near the minimum of the solution in order to avoid premature or "false" singularities [27, 25].

For the computation of nonnegative weak solutions, a non-negativity preserving finite element method is proposed in [8]. Non-negativity of the solution is imposed as a constraint, so we have to solve a variational problem involving a Lagrange multiplier at every time step to advance the solution. Another non-negativity preserving scheme, that has some common elements to the work discussed here, is introduced by Grün and Rumpf [56]. The method presented here is from a joint paper by L. Zhornitskaya with the first author [103]. 
This section presents results from [103], illustrating that it is possible to design a finite difference (in space) scheme to satisfy discrete analogues of properties (12)-(14) above. Such a scheme preserves positivity of the solution (whenever $n \geq 2$ ) and has solutions that exist for all time, regardless of the size of the grid. This method improves upon previous methods (e.g. [27]) that require mesh refinement in order to avoid a premature 'numerical' singularity. We show that it is possible to generalize the positivity preserving property to finite element methods on arbitrary element spaces (including those involving nonuniform grids).

\subsection{The solution of the difference equations}

We consider a family of continuous-time, discrete-space finite difference schemes for the one dimensional lubrication equation (7) with periodic boundary conditions and strictly positive initial data $h_{0}(x) \in H^{1}\left(S^{1}\right)$.

Notation: Divide the periodic domain $S^{1}$ into N equally spaced regions of length $\Delta x$ and introduce the following discrete analogues of the space derivatives: ${ }^{5}$

$$
\begin{aligned}
& y_{x, i}=\frac{y_{i+1}-y_{i}}{\Delta x}, \quad y_{\bar{x}, i}=\frac{y_{i}-y_{i-1}}{\Delta x}, \\
& y_{\bar{x} x, i}=\frac{y_{\bar{x}, i+1}-y_{\bar{x}, i}}{\Delta x}, \quad y_{\bar{x} x \bar{x}, i}=\frac{y_{\bar{x} x, i}-y_{\bar{x} x, i-1}}{\Delta x} .
\end{aligned}
$$

The discrete $H_{1, \Delta x}$ norm is $\|y\|_{1, \Delta x}=\left(\sum_{i}\left(y_{\bar{x}, i}^{2}+y_{i}^{2}\right) \Delta x\right)^{1 / 2}$ which is equivalent to $\left(\left(\sum_{i} y_{\bar{x}, i}^{2} \Delta x\right)+\bar{y}^{2}\right)^{1 / 2}$, where $\bar{y}$ denotes the mean $\sum_{i} y_{i} \Delta x$. The numerical scheme

$$
\begin{aligned}
& y_{i, t}+\left(a\left(y_{i-1}, y_{i}\right) y_{\bar{x} x \bar{x}, i}\right)_{x}=0, \\
& i=0,1, \ldots, N-1, \\
& y_{i}(0)=h_{0}\left(x_{i}\right),
\end{aligned}
$$

which we can view as a coupled system of ordinary differential equations for the $y_{i}$, is a continuous-time discrete-space approximation of the PDE (7) provided that the nonlinear coefficient $a\left(s_{1}, s_{2}\right)$ satisfies the following definition:

Definition 2.1 (Generic finite difference scheme)

$$
\begin{aligned}
& a(s, s)=f(s), \\
& a\left(s_{1}, s_{2}\right)=a\left(s_{2}, s_{1}\right), \\
& a\left(s_{1}, s_{2}\right) \in C^{4}((0, \infty) \times(0, \infty)) \cap C([0, \infty) \times[0, \infty)), \\
& \forall \delta>0, \text { there exists } \gamma>0 \text { such that } s_{1}, s_{2}>\delta \Longrightarrow a\left(s_{1}, s_{2}\right) \geq \gamma>0 .
\end{aligned}
$$

Conditions (a)-(c) guarantee the scheme (16) is second order consistent with a positive smooth solution of (7). Condition (c) does not require $a\left(s_{1}, s_{2}\right)$ to be differentiable at the origin. Condition (d) says that $a\left(s_{1}, s_{2}\right)$ is positive whenever both its arguments are positive, but may become degenerate if at least one of its arguments approaches zero. This matches

\footnotetext{
${ }^{5}$ We append the ,i on to the $x$ and $\bar{x}$ subscripts to make it clear that we are computing a finite difference as opposed to a spatial derivative. Throughout the rest of the paper, we use $\partial_{x}, \nabla$, etc. to denote space derivatives, and subscripts to denote finite differences in space.
} 
the property of the analytical solution (7) since $f(h) \sim h^{n}$ is positive whenever $h$ is positive but $f(h) \rightarrow 0$ as $h \rightarrow 0$. Both $a\left(s_{1}, s_{2}\right)=f\left(\frac{1}{2}\left(s_{1}+s_{2}\right)\right)$ and $a\left(s_{1}, s_{2}\right)=\frac{1}{2}\left(f\left(s_{1}\right)+f\left(s_{2}\right)\right)$ are examples of allowed discretizations. The former discretization is used, for example, in [27].

The following properties of the numerical scheme are analogues of the continuous case:

- Discrete conservation of mass.

$$
\sum_{i=0}^{N-1} y_{i}(t) \Delta x=\sum_{i=0}^{N-1} y_{i}(0) \Delta x
$$

- Discrete energy dissipation.

$$
\frac{1}{2} \sum_{i}\left(y_{\bar{x}, i}(t)\right)^{2} \Delta x+\int_{0}^{t} \sum_{i} a\left(y_{i-1}(s), y_{i}(s)\right)\left(y_{\bar{x} x \bar{x}, i}(s)\right)^{2} \Delta x d s=\frac{1}{2} \sum_{i}\left(y_{\bar{x}, i}(0)\right)^{2} \Delta x .
$$

Standard ODE arguments allow us to prove the following lemma.

Lemma 2.2 (Existence, uniqueness and continuation of solutions of the numerical scheme [103]) Given the coupled ODE system (16) with strictly positive initial data $y_{i}(0)$ and diffusion coefficient $a\left(s_{1}, s_{2}\right)$ satisfying conditions(a)-(d) from Definition 2.1, there is a time interval $[0, \sigma]$ for which there exists a unique positive solution of the coupled ODE system (16). Moreover, there exists a constant $C$, depending only on the discrete $H_{1, \Delta x}$ norm of the initial data $y_{i}(0)$, such that $y_{i}(t) \leq C$ for all $i$ and all $t \leq \sigma$. The solution can be continued for arbitrarily long times provided it remains positive.

\subsection{A positivity preserving finite difference scheme}

In this section we show that if $n \geq 2$ in (8) then there exists a way to choose the function $a\left(s_{1}, s_{2}\right)$ in (16) so that, for positive initial data, the solution of (16) stays positive for all time. This property is a discrete analogue of the weak maximum principle for the PDE (8). Our goal is to choose $a\left(s_{1}, s_{2}\right)$ so that the numerical scheme (16) satisfies a discrete form of (14) and show that this is sufficient to guarantee that its solutions also preserve positivity.

Lemma 2.3 [103] Let $G^{\prime \prime}(v)=\frac{1}{f(v)}$ be a nonlinear entropy function. If we choose $a\left(s_{1}, s_{2}\right)$ in (16) to satisfy

$$
a\left(s_{1}, s_{2}\right)= \begin{cases}\frac{s_{1}-s_{2}}{G^{\prime}\left(s_{1}\right)-G^{\prime}\left(s_{2}\right)} & \text { if } s_{1} \neq s_{2} \\ f\left(s_{1}\right) & \text { if } s_{1}=s_{2}\end{cases}
$$

then the solution of (16), with positive initial data, satisfies the following discrete entropy dissipation property

$$
\sum_{i} G\left(y_{i}(t)\right) \Delta x+\int_{0}^{t}\left\{\sum_{i}\left(y_{\bar{x} x, i}(s)\right)^{2} \Delta x\right\} d s=\sum_{i} G\left(y_{i}(0)\right) \Delta x .
$$


This leads to the following proposition showing that the scheme results in a positive solution regardless of the grid size.

Proposition 2.4 Let $f(h) \sim h^{n}$, as $h \rightarrow 0, n \geq 2$ and

$$
a\left(s_{1}, s_{2}\right)= \begin{cases}\frac{s_{1}-s_{2}}{G^{\prime}\left(s_{1}\right)-G^{\prime}\left(s_{2}\right)} & \text { if } s_{1} \neq s_{2}, \\ f\left(s_{1}\right) & \text { if } s_{1}=s_{2} .\end{cases}
$$

Then given positive initial data $y_{i}(0)>0$, for every fixed $\Delta x$ there exists $\bar{\delta}>0$ such that $\min _{t} \min _{i} y_{i}(t) \geq \bar{\delta}$. Moreover, if $n \geq 4$ then $\bar{\delta}$ depends only on $\left\|y_{i}\right\|_{1, \Delta x}$ and $\min _{i} y_{i}$. In particular, it does not depend directly on $\Delta x$.

Using the discrete entropy dissipation property (20), we get

$$
\begin{gathered}
\sum_{j} \frac{1}{y_{j}^{n-2}} \Delta x \leq \tilde{C}, \text { if } n>2, \\
\sum_{j} \ln \left(\frac{1}{y_{j}}\right) \Delta x \leq \tilde{C}, \text { if } n=2,
\end{gathered}
$$

where $\tilde{C}$ depends on the uniform positive lower and (for $n=2$ ) upper bounds of the initial condition $y_{i}(0)$. This implies that

$$
\begin{gathered}
\min _{t} \min _{i} y_{i}(t) \geq\left(\frac{\Delta x}{\tilde{C}}\right)^{\frac{1}{n-2}} \equiv \bar{\delta}, \text { if } n>2, \\
\min _{t} \min _{i} y_{i}(t) \geq \exp \left(-\frac{\tilde{C}}{\Delta x}\right) \equiv \bar{\delta}, \text { if } n=2 .
\end{gathered}
$$

This estimate is valid for any $n \geq 2$. However, it depends badly on $\Delta x$, that is, as $\Delta x$ gets smaller the lower bound gets smaller as well. Since there exists a positive lower bound on the solution of the PDE (8) for $n$ large enough, we would like to obtain a lower bound independent of $\Delta x$ for the discrete case as well. The following argument, similar to that for the continuous case [13], shows that this is indeed possible for $n \geq 4$.

The discrete energy dissipation property and the Sobolev Lemma imply the existence of an upper bound on the discrete $C_{\Delta x}^{1 / 2}$ of $y_{i}(t)$. Let $\delta(t)=\min _{0 \leq i \leq N-1} y_{i}(t)$, which occurs at $i(t)$. Then we get

$$
\begin{aligned}
& \tilde{C} \geq \sum_{j} \frac{1}{y_{j}^{n-2}} \Delta x \geq \sum_{j} \frac{\Delta x}{\left(\delta(t)+C|(j-i(t)) \Delta x|^{1 / 2}\right)^{n-2}} \geq \\
& 2 \frac{1}{\delta^{n-4}} \int_{0}^{1 / \delta^{2}} \frac{d s}{\left(1+s^{1 / 2}\right)^{n-2}} .
\end{aligned}
$$

Now to obtain a bound independent of $\Delta x$, we consider two cases: $\delta>1$ and $\delta \leq 1$. In the former case we already have the bound we wanted. In the latter case we get

$$
\begin{aligned}
& \tilde{C} \geq 2 \frac{1}{\delta^{n-4}} \int_{0}^{1 / \delta^{2}} \frac{d s}{\left(1+s^{1 / 2}\right)^{n-2}} \geq 2 \frac{1}{\delta^{n-4}} \int_{0}^{1} \frac{d s}{\left(1+s^{1 / 2}\right)^{n-2}}=\frac{C^{\prime}}{\delta^{n-4}}, \text { if } n>4, \\
& \tilde{C} \geq 2 \int_{0}^{1 / \delta^{2}} \frac{d s}{\left(1+s^{1 / 2}\right)^{2}} \geq C^{\prime} \ln \left(\frac{1}{\delta^{2}}\right), \quad \text { if } n=4,
\end{aligned}
$$


which again implies the existence of a lower bound independent of $\Delta x$.

So, with the choice of spatial discretization (21) the finite difference scheme (16) is positivity preserving for all $n \geq 2$, and the lower bound is independent of $\Delta x$ provided $n \geq 4$.

Note that the above results, coupled with Lemma 2.2, prove global existence of positive solutions of the scheme (16) with the special choice of $a\left(s_{1}, s_{2}\right)$ in $(21)$. We state this as a corollary.

Corollary 2.5 Let $f(h) \sim h^{n}$ with $n \geq 2$. The finite difference scheme (16), with the special choice of $a\left(s_{1}, s_{2}\right)$ satisfying (21), and positive initial data $y_{i}(0)=h_{0}\left(x_{i}\right)>0$ has a global in time positive solution $y_{i}(t)$ regardless of the size of the mesh $\Delta x$.

\subsection{Consistency and convergence}

To measure consistency, we introduce the local truncation error $\tau_{i}(t)$, defined as the result of substituting the solution of the PDE (8) into (16):

$$
h_{i, t}+\left(a\left(h_{i-1}, h_{i}\right) h_{\bar{x} x \bar{x}, i}\right)_{x}=\tau_{i}(t) .
$$

Since a positive solution of (7) is infinitely differentiable [13], we can use Taylor series expansions to examine the consistency of the numerical scheme.

Lemma 2.6 [103] Let a $\left(s_{1}, s_{2}\right)$ satisfy the conditions (a)-(d) of Definition 2.1 and let $h(x, t)$ be a smooth positive solution of (7). Then the local truncation error $\tau_{i}(t)$ in (22) is $O\left((\Delta x)^{2}\right)$ uniformly in $t$.

The error depends on the size of the higher derivatives of $h$, which are known empirically $[27,25]$ to become unbounded when a positive solution approaches zero.

If the grid size is too large, a generic scheme may fail to have a solution for all time due to numerical singularities. In the case of a generic scheme existence of a solution is guaranteed only for a sufficiently small grid size.

Theorem 2.7 [103] Let $h(x, t)$ be a smooth solution of (7) such that $h(x, t) \geq \delta>0$ for some $\delta>0$ and $t \in[0, T]$. Then for $\Delta x$ sufficiently small there exists a unique solution $y_{i}(t)$ of (16) for all $t \in[0, T]$. Moreover, there exists a constant $C>0$ such that $\sup _{t \in[0, T]} \|$ $y_{i}(t)-h\left(x_{i}, t\right) \|_{1, \Delta x} \leq C \Delta x^{2}$.

This convergence result includes the positivity preserving scheme which has solutions even on very coarse grids.

\subsection{Modified entropy dissipating scheme}

In Corollary 2.5 we showed that if $n \geq 2$ then for all $\Delta x$ there exists a unique positive solution of the entropy dissipating scheme (EDS) for all $t$. However, for $n<2$ the EDS scheme (16), (21) may not be positivity preserving. In examples such as the Hele-Shaw cell ((8) with $n=1$ ), we may wish to use a positivity preserving scheme to approximate a positive solution. We now present a modification of the EDS that yields a positivity preserving scheme for all 
$n>0$. To accomplish this, we use the following regularization of the PDE (7), introduced in $[13]$

$$
h_{\epsilon t}+\partial_{x}\left(f_{\epsilon}\left(h_{\epsilon}\right) \partial_{x}^{3} h_{\epsilon}\right)=0, f_{\epsilon}\left(h_{\epsilon}\right)=\frac{h_{\epsilon}^{4} f\left(h_{\epsilon}\right)}{\epsilon f\left(h_{\epsilon}\right)+h_{\epsilon}^{4}},
$$

and the corresponding numerical scheme

$$
\begin{aligned}
& y_{\epsilon, i, t}+\left(a_{\epsilon}\left(y_{\epsilon, i-1}, y_{\epsilon, i}\right) y_{\epsilon \bar{x} x \bar{x}, i}\right)_{x}=0, \\
& i=0,1, \ldots, N-1, \\
& y_{\epsilon, i}(0)=h_{0}\left(x_{i}\right), \\
& a_{\epsilon}\left(s_{1}, s_{2}\right)= \begin{cases}\frac{s_{1}-s_{2}}{G_{\epsilon}^{\prime}\left(s_{1}\right)-G_{\epsilon}^{\prime}\left(s_{2}\right)} & \text { if } s_{1} \neq s_{2}, \\
f_{\epsilon}\left(s_{1}\right) & \text { if } s_{1}=s_{2} .\end{cases}
\end{aligned}
$$

Note that numerical method (23)-(24) depends on two independent parameters, namely $\Delta x$ and $\epsilon$. However we could choose $\epsilon=\epsilon(\Delta x)$ to be a function of the grid size.

Since $f_{\epsilon}\left(h_{\epsilon}\right) \sim \frac{h_{\epsilon}^{4}}{\epsilon}$ as $h_{\epsilon} \rightarrow 0$ we know that for all $\epsilon>0$ and $\Delta x>0$ the solution of (23)(24) is positive. Moreover, the following theorem shows that the solution of the modified EDS converges to the solution of the original (nonregularized) PDE as $\epsilon \rightarrow 0$ and $\Delta x \rightarrow 0$ as long as the solution of the latter stays positive. The proof relies on the fact that for all $\bar{\delta}>0, m, n \in N$, there exists a constant $C(m, n, \bar{\delta})$ such that

$$
\left\|\frac{\partial^{n+m}}{\partial s_{1}^{n} \partial s_{2}^{m}} a_{\epsilon}\left(s_{1}, s_{2}\right)-\frac{\partial^{n+m}}{\partial s_{1}^{n} \partial s_{2}^{m}} a\left(s_{1}, s_{2}\right)\right\|_{L^{\infty}\left([\bar{\delta}, M]^{2}\right)} \leq C(m, n, \bar{\delta}) \epsilon .
$$

Theorem 2.8 Let $h(x, t)$ be a smooth solution of (8) such that $h(x, t) \geq \delta>0$ for some $\delta>0$ and $t \in[0, T]$. Then for all $\Delta x$ and $\epsilon$ there exists a unique solution $y_{\epsilon, i}(t)$ of the modified $E D S$ (23)-(24) for all $t \in[0, T]$. Moreover, there exists a constant $C>0$ independent of $\Delta x$ and $\epsilon$ such that

$$
\sup _{t \in[0, T]}\left\|y_{\epsilon, i}(t)-h\left(x_{i}, t\right)\right\|_{1, \Delta x} \leq C\left(\Delta x^{2}+\epsilon\right) .
$$

We can then choose $\epsilon \sim(\Delta x)^{2}$ to make the scheme (23)-(24) second order in $\Delta x$.

\subsection{Finite difference schemes in 2D}

In two space dimensions the lubrication equation with periodic boundary conditions is

$$
\begin{aligned}
& h_{t}+\partial_{x}\left(f(h) \partial_{x}\left(\partial_{x}^{2} h+\partial_{y}^{2} h\right)\right)+ \\
& \quad \partial_{y}\left(f(h) \partial_{y}\left(\partial_{x}^{2} h+\partial_{y}^{2} h\right)\right)=0, x \in S^{1}, y \in S^{1} .
\end{aligned}
$$

Let $z_{i j}(t)$ be a solution of the following finite difference scheme

$$
\begin{aligned}
z_{i, j, t}+ & \left(a\left(z_{i-1, j}, z_{i, j}\right)\left(z_{\bar{x} x, i j}+z_{\bar{y} y, i j}\right)_{\bar{x}}\right)_{x}+ \\
\left(a\left(z_{i, j-1}, z_{i, j}\right)\left(z_{\bar{x} x, i j}+z_{\bar{y} y, i j}\right)_{\bar{y}}\right)_{y} & =0,
\end{aligned}
$$

where $a\left(s_{1}, s_{2}\right)$ is an approximation of $f(h)$ satisfying Definition 2.1. 
As in the one-dimensional case, consider the finite difference scheme (27) as a coupled system of ODEs. Given positive initial data, there exists, locally in time, a unique solution of (27); the proof follows from the same arguments as in Lemma 2.2. Moreover, following the argument there, we can uniquely continue the solution in time provided it stays bounded away from zero and infinity. Therefore, a unique solution exists globally whenever we can prove the existence of a priori upper and lower bounds.

A generic finite difference scheme (27) has the following properties:

- Discrete conservation of mass.

$$
\begin{gathered}
\sum_{i=0}^{N-1} \sum_{j=0}^{M-1} z_{i j, t} \Delta x \Delta y+\sum_{i=0}^{N-1} \sum_{j=0}^{M-1}\left(a\left(z_{i-1, j}, z_{i, j}\right)\left(z_{\bar{x} x, i j}+z_{\bar{y} y, i j}\right)_{\bar{x}}\right)_{x} \Delta x \Delta y+ \\
\sum_{i=0}^{N-1} \sum_{j=0}^{M-1}\left(a\left(z_{i, j-1}, z_{i, j}\right)\left(z_{\bar{x} x, i j}+z_{\bar{y} y, i j}\right)_{\bar{y}}\right)_{y} \Delta x \Delta y=0 \\
\text { or equivalently } \\
\sum_{i=0}^{N-1} \sum_{j=0}^{M-1} z_{i j}(t) \Delta x \Delta y=\sum_{i=0}^{N-1} \sum_{j=0}^{M-1} z_{i j}(0) \Delta x \Delta y .
\end{gathered}
$$

- Discrete energy dissipation.

Multiplication of $(27)$ by $\left(z_{\bar{x} x, i j}+z_{\bar{y} y, i j}\right) \Delta x \Delta y$, summation over $i, j$ and integration in time gives

$$
\begin{aligned}
& \frac{1}{2} \sum_{i, j}\left\{\left(z_{\bar{x}, i j}(t)\right)^{2}+\left(z_{\bar{y}, i j}(t)\right)^{2}\right\} \Delta x \Delta y+ \\
& \int_{0}^{t}\left\{\sum_{i, j} a\left(z_{i-1, j}(s), z_{i j}(s)\right)\left(z_{\bar{x} x, i j}(s)+z_{\bar{y} y, i j}(s)\right)_{\bar{x}}^{2} \Delta x \Delta y\right\} d s+ \\
& \int_{0}^{t}\left\{\sum_{i, j} a\left(z_{i, j-1}(s), z_{i j}(s)\right)\left(z_{\bar{x} x, i j}(s)+z_{\bar{y} y, i j}(s)\right)_{\bar{y}}^{2} \Delta x \Delta y\right\} d s= \\
& \frac{1}{2} \sum_{i, j}\left\{\left(z_{\bar{x}, i j}(0)\right)^{2}+\left(z_{\bar{y}, i j}(0)\right)^{2}\right\} \Delta x \Delta y .
\end{aligned}
$$

The above mass conservation and energy dissipation combine to give the following estimate:

Lemma 2.9 There exists a constant $C$ such that for any $z_{i j}$ a positive solution of (27), on the time interval $0 \leq t \leq T$, with initial data $z_{i j}(0)=h_{0}\left(x_{i}, y_{j}\right)>0$, we have the a priori upper bound

$$
z_{i j}(t) \leq \frac{C\left\|z_{i j}(0)\right\|_{1, \Delta x, \Delta y}}{\sqrt{\Delta x \Delta y}}
$$

where $\|\cdot\|_{1, \Delta x, \Delta y}$ denotes the discrete $H^{1}$ norm in $2 D$. 
We now construct a special entropy dissipating scheme that gives an a priori pointwise lower bound. The following lemma shows that the same discretization of the diffusion coefficient that gives dissipation of the nonlinear entropy in $1 \mathrm{D}$ also works in 2D.

Lemma 2.10 Let $G^{\prime \prime}(v)=\frac{1}{f(v)}$ be a nonlinear entropy function. Let $f(h) \sim h^{n}$ as $h \rightarrow 0$. Then if we choose

$$
a\left(s_{1}, s_{2}\right)= \begin{cases}\frac{s_{1}-s_{2}}{G^{\prime}\left(s_{1}\right)-G^{\prime}\left(s_{2}\right)}, & \text { if } s_{1} \neq s_{2} \\ f\left(s_{1}\right), & \text { if } s_{1}=s_{2}\end{cases}
$$

then any solution of (27) with positive initial data $z_{i j}(0)=h_{0}\left(x_{i}, y_{j}\right)>0$ satisfies the following discrete entropy dissipation property

$$
\begin{aligned}
& \sum_{i, j} G\left(z_{i j}(t)\right) \Delta x \Delta y+\int_{0}^{t}\left\{\sum_{i, j}\left(z_{\bar{x} x, i j}(s)+z_{\bar{y} y, i j}(s)\right)^{2} \Delta x \Delta y\right\} d s= \\
& \sum_{i, j} G\left(z_{i j}(0)\right) \Delta x \Delta y .
\end{aligned}
$$

This property implies that the solution $z_{i j}(t)$ has the following pointwise lower bound

$$
\begin{gathered}
\min _{t} \min _{i j} z_{i j}(t) \geq\left(\frac{\Delta x \Delta y}{\tilde{C}}\right)^{\frac{1}{n-2}} \equiv \bar{\delta}, \quad \text { if } n>2, \\
\min _{t} \min _{i j} z_{i j}(t) \geq \exp \left(-\frac{\tilde{C}}{\Delta x \Delta y}\right) \equiv \bar{\delta}, \quad \text { if } n=2 .
\end{gathered}
$$

In $2 \mathrm{D}$ we do not have a uniform lower bound independent of the grid size for large values of $n$ as we did in the $1 \mathrm{D}$ case. This is due to the fact that the $1 \mathrm{D}$ argument used the a priori $C^{1 / 2}$ bound, from Sobolev embedding, which we do not know for the $2 \mathrm{D}$ case. The fact that we can derive an a priori pointwise lower bound implies the following corollary:

Corollary 2.11 (Global existence of solutions of the 2D entropy dissipating scheme) Consider $f(h) \sim h^{n}$ as $h \rightarrow 0$, for $n \geq 2$. Then for all $\Delta x, \Delta y$, the entropy dissipating scheme (27) with $a\left(s_{1}, s_{2}\right)$ satisfying (31) and positive initial data $z_{i j}(0)=h_{0}\left(x_{i}, y_{j}\right)>0$ has a unique positive global in time solution.

In general the generic scheme (27) may only have local solutions in time. The issue is that the solution may lose positivity at some finite time and can not be continued after that time. In addition we have the following convergence result.

Theorem 2.12 (Convergence) Let $h(x, y, t)$ be a smooth solution of (26) such that $C \geq$ $h(x, y, t) \geq \delta>0$ for some $C>\delta>0$ and $t \in[0, T]$. Let $z_{i j}(t)$ be a solution of the numerical scheme (27) with $(\Delta x)^{3} \ll \Delta y \ll(\Delta x)^{1 / 3}$. Then for $\Delta x, \Delta y$ sufficiently small the solution $z_{i j}(t)$ can be continued for all $t \in[0, T]$ and there exists a constant $C>0$ such that we have the $H^{1}$ convergence

$$
\sup _{t \in[0, T]}\left\|z_{i j}(t)-h\left(x_{i}, y_{j}, t\right)\right\|_{1, \Delta x, \Delta y} \leq C\left(\Delta x^{2}+\Delta y^{2}\right)
$$


and the pointwise convergence

$$
\sup _{t \in[0, T]} \sup _{i, j}\left|z_{i j}(t)-h\left(x_{i}, y_{j}, t\right)\right|^{2} \leq C \frac{\left((\Delta x)^{2}+(\Delta y)^{2}\right)^{2}}{\Delta x \Delta y} .
$$

\subsection{Positivity preserving finite element methods}

A natural framework is that of finite elements. We begin by showing that the 1D EDS ((16) with (21)) finite difference scheme is equivalent to a finite element approximation in which a nonlinear function of the solution is represented in the element basis.

Example 2.13 (1D EDS) First introduce the pressure $p=-\partial_{x}^{2} h$. The original PDE

$$
h_{t}+\partial_{x}\left(f(h) \partial_{x}^{3} h\right)=0
$$

can be rewritten as

$$
h_{t}-\partial_{x}\left(f(h) \partial_{x} p\right)=0, p=-\partial_{x}^{2} h .
$$

Let $\left(\eta_{1}, \eta_{2}\right)$ denote the standard inner product on $L^{2}\left(S^{1}\right), G(y)$ be a function satisfying $G^{\prime \prime}(y)=1 / f(y), T^{\Delta x}$ be a space of piecewise linear periodic functions on the spatial grid of size $\Delta x$. We introduce the interpolation operator $\pi^{\Delta x}: C\left(S^{1}\right) \rightarrow T^{\Delta x}$ such that $\left(\pi^{\Delta x} \eta\right)\left(x_{j}\right)=$ $\eta\left(x_{j}\right)$ for all $j$. Define a discrete inner product on $C\left(S^{1}\right)$ by

$$
\left(\eta_{1}, \eta_{2}\right) \stackrel{\Delta x}{\Delta} \stackrel{\text { def }}{=} \int_{S^{1}} \pi^{\Delta x}\left(\eta_{1}(x) \eta_{2}(x)\right) d x \equiv \sum_{j} \eta_{1}\left(x_{j}\right) \eta_{2}\left(x_{j}\right) \Delta x .
$$

The EDS is equivalent to the following finite element approximation of (36):

Find $y, w: G^{\prime}(y) \in T^{\Delta x}, w \in T^{\Delta x}$ such that

$$
\begin{aligned}
\left(y_{t}, \chi\right)^{\Delta x}+\left(f(y) \partial_{x} w, \partial_{x} \chi\right)=0 & \forall \chi \in T^{\Delta x} \\
\left(\partial_{x} y, \partial_{x} \chi\right)=(w, \chi)^{\Delta x} & \forall \chi \in T^{\Delta x}
\end{aligned}
$$

Consider the standard basis $\left\{\chi_{i}(x)\right\}_{i=1}^{N}$ for $T^{\Delta x}$ :

$$
\chi_{i}(x)= \begin{cases}\frac{x-x_{i-1}}{\Delta x} & \text { if } x_{i-1} \leq x \leq x_{i} \\ \frac{x_{i+1}-x}{\Delta x} & \text { if } x_{i} \leq x \leq x_{i+1}\end{cases}
$$

Substitution in (37-38) gives $\left(y_{t}, \chi_{i}\right)^{\Delta x}=y_{i, t} \Delta x$ and

$$
y_{i, t}+\frac{1}{\Delta x}\left(y_{\bar{x} x \bar{x}, i+1} \frac{y_{i+1}-y_{i}}{G^{\prime}\left(y_{i+1}\right)-G^{\prime}\left(y_{i}\right)}-y_{\bar{x} x \bar{x}, i} \frac{y_{i}-y_{i-1}}{G^{\prime}\left(y_{i}\right)-G^{\prime}\left(y_{i-1}\right)}\right)=0,
$$

which indeed is the EDS (16) with (19). 
The same finite element approximation on a non-uniform mesh produces an analogous scheme (39) with nonuniform differences. A variant of this scheme is used in [22] to compute finite time singularities in a long-wave unstable generalization of (35).

The finite element representation (37-38) has similar structure to that introduced in [8]. The main difference is that the method in [8] takes the solution $y$ to be in the subspace $T^{\Delta x}$ spanned by the element basis, resulting in a method that requires a Lagrange multiplier to insure nonnegativity of the solution in cases where it might otherwise become negative. Here, we take the nonlinear function $G^{\prime}(y) \in T^{\Delta x}$, resulting in a scheme that preserves positivity. Our choice of element representation has one consequence: we need to insure that $\nabla y \in L^{2}$ in order to make sense of the inner product on the left hand side of (38).

The finite element approach above generalizes to positivity preserving schemes in higher dimensions $\left(S^{1}\right)^{d}$ and to more complicated finite element subspaces of $H^{1}\left(\left(S^{1}\right)^{d}\right)$. Consider the following form of the lubrication equation in $d$ space dimensions:

$$
h_{t}-\nabla \cdot(f(h) \nabla p)=0, \quad p+\Delta h=0, \quad f(h) \sim h^{n}, h \rightarrow 0 .
$$

Now let $\left(\eta_{1}, \eta_{2}\right)$ denote an inner product in $L^{2}\left(\left(S^{1}\right)^{d}\right), T^{\Delta \vec{x}}$ be a finite dimensional subspace of $H^{1}\left(\left(S^{1}\right)^{d}\right), \pi^{I_{1}}: C\left(\left(S^{1}\right)^{d}\right) \rightarrow T^{\Delta \vec{x}}$ be an interpolation operator and $\left(\eta_{1}, \eta_{2}\right)^{I_{1}} \stackrel{\text { def }}{=}$ $\int_{\left(S^{1}\right)^{d}} \pi^{I_{1}}\left(\eta_{1}(x) \eta_{2}(x)\right) d x$, be an associated inner product on $C\left(\left(S^{1}\right)^{d}\right)$. For any $d$-dimensional vectors $\xi_{1}, \xi_{2} \in C\left(\left(S^{1}\right)^{d}, R^{d}\right)$ let $\left(\xi_{1}, \xi_{2}\right)^{I_{2}}$ denote a numerical integration rule replacing an $L^{2}\left(\left(S^{1}\right)^{d}, R^{d}\right)$ inner product.

Consider the following general finite element approximation of (40).

Find $z, w: G^{\prime}(z) \in T^{\Delta \vec{x}}, w \in T^{\Delta \vec{x}}$ such that

$$
\begin{array}{r}
\left(z_{t}, \chi\right)^{I_{1}}+(f(z) \nabla w, \nabla \chi)^{I_{2}}=0, \quad \forall \chi \in T^{\Delta \vec{x}}, \\
(\nabla z, \nabla \chi)^{I_{2}}=(w, \chi)^{I_{1}}, \quad \forall \chi \in T^{\Delta \vec{x}} .
\end{array}
$$

Taking $\chi=G^{\prime}(z)$ in (41) and $\chi=w$ in (42), we obtain

$$
\begin{array}{r}
\left(z_{t}, G^{\prime}(z)\right)^{I_{1}}+(\nabla w, \nabla z)^{I_{2}}=0, \\
(\nabla z, \nabla w)^{I_{2}}=(w, w)^{I_{1}},
\end{array}
$$

or

$$
\frac{d}{d t} \int_{\left(S^{1}\right)^{d}} \pi^{I_{1}}(G(z)) d x=-(w, w)^{I_{1}} \leq 0
$$

This yields the following a priori bound:

$$
\int_{\left(S^{1}\right)^{d}} \pi^{I_{1}}(G(z(x, t))) d x \leq C .
$$

Now by using an explicit form of the interpolation operator $\pi^{I_{1}}$ we can rewrite the left hand side of the above inequality as $\sum_{i} a_{i} G\left(z_{i}(t)\right)$ with $a_{i}>0$ for all $i$. As before, this gives a positive (dependent on $\Delta \vec{x}$ ) lower bound on $z_{i}(t)$ for $n \geq 2$.

We have to insure that $\nabla z \in L^{2}\left(\left(S^{1}\right)^{d}, R^{d}\right)$ to use the finite element method (41)-(42). This is guaranteed as long as all $z_{i}(t)$ are bounded from above. In the special cases of the 
above example and the next example below, additional structure of the scheme allows us to prove an a priori bound on the discrete $H^{1}$ norm of the solution $z$, independent of the grid size. In general, however, discrete energy dissipation (analogous to (18)) may not occur for (41-42). Note that since $G^{\prime}(z) \in T^{\Delta \vec{x}}$, in general $\nabla G^{\prime}(z)=\frac{1}{f(z)} \nabla z \in L^{2}\left(\left(S^{1}\right)^{d}\right)$. Thus a sufficient condition for $\nabla z \in L^{2}\left(\left(S^{1}\right)^{d}, R^{d}\right)$ is that $f(z)$ is bounded. This is also a sufficient condition for $f(z) \nabla w \in L^{2}\left(\left(S^{1}\right)^{d}, R^{d}\right)$ which is needed to make sense of the nonlinear term in (41).

Now let us consider some further examples of the general finite element method (41-42).

Example 2.14 In two space dimensions, let $\vec{x}=(x, y)$ and $T^{\Delta \vec{x}}$ be a space of piecewise bilinear functions on the rectangles of size $\Delta x \times \Delta y$. Let $\chi_{i j}(x, y)$ be a basis for this space such that $\chi_{i j}(x, y)$ is equal to 1 at node $\left(x_{i}, y_{j}\right)$ and 0 at all other nodes. As before, let $\pi^{I_{1}}$ : $C\left(\left(S^{1}\right) 2\right) \rightarrow T^{\Delta \vec{x}}$ be the interpolation operator such that for any continuous function $\eta(x, y)$ $\left(\pi^{I_{1}} \eta\right)\left(x_{i}, y_{j}\right)=\eta\left(x_{i}, y_{j}\right)$ for all $i, j$. For any 2 -dimensional vectors $\xi_{1}, \xi_{2} \in C\left(\left(S^{1}\right)^{2}, R^{2}\right)$ let

$$
\begin{aligned}
\left(\xi_{1}, \xi_{2}\right)^{I_{2}=} & \sum_{i, j} \int_{x_{i}}^{x_{i+1}} \frac{1}{2}\left(\xi_{1}^{1}\left(x, y_{j+1}\right) \xi_{2}^{1}\left(x, y_{j+1}\right)+\xi_{1}^{1}\left(x, y_{j}\right) \xi_{2}^{1}\left(x, y_{j}\right)\right) \Delta y d x+ \\
& \sum_{i, j} \int_{y_{j}}^{y_{j+1}} \frac{1}{2}\left(\xi_{1}^{2}\left(x_{i+1}, y\right) \xi_{2}^{2}\left(x_{i+1}, y\right)+\xi_{1}^{2}\left(x_{i}, y\right) \xi_{2}^{2}\left(x_{i}, y\right)\right) \Delta x d y
\end{aligned}
$$

On the product of first components this numerical integration rule performs integration exactly in the first variable, but uses the trapezoidal rule instead of integration in the second variable. Similarly, on the product of second components it performs integration exactly in the second variable, but uses the trapezoidal rule instead of integration in the first variable. We now show that this choice gives us the $2 D$ positivity preserving scheme (27) with (31) from Section 2.5. Taking $\chi=\chi_{i j}$ in (41) gives $\left(z_{t}, \chi_{i j}\right)^{I_{1}}=z_{i j, t} \Delta x \Delta$ yand

$$
z_{i j, t}+\left(a\left(z_{i-1, j}, z_{i, j}\right)\left(z_{\bar{x} x, i j}+z_{\bar{y} y, i j}\right)_{\bar{x}}\right)_{x}+\left(a\left(z_{i, j-1}, z_{i, j}\right)\left(z_{\bar{x} x, i j}+z_{\bar{y} y, i j}\right)_{\bar{y}}\right)_{y}=0,
$$

which is the positivity preserving $2 D$ scheme ((27) and (31)) that we introduced in Section 2.5.

In previous sections on finite differences we encountered both of the examples considered above. Now we introduce a new scheme that arises from a different choice of inner products in $(41-42)$.

Example 2.15 In $1 D$ consider the same scheme as (37)-(38) except with trapezoidal rule instead of exact integration for computation of $\left(f(y) \partial_{x} w, \partial_{x} \chi\right)$ and $\left(\partial_{x} y, \partial_{x} \chi\right)$. Then $\left(z_{t}, \chi_{i}\right)^{I_{1}}=$ $z_{i, t} \Delta x$ and we obtain the scheme

$$
z_{i, t}-\left(a\left(z_{i-1}, z_{i}\right) w_{\bar{x}, i}\right)_{x}=0
$$

with

$$
\begin{aligned}
& a\left(z_{i-1}, z_{i}\right)=\frac{f\left(z_{i-1}\right)+f\left(z_{i}\right)}{2} \quad \text { and } \\
& w_{i}=-\left(\left\{G^{\prime}\left(z_{i}\right)\right\}_{\bar{x}} a\left(z_{i-1}, z_{i}\right)\right)_{x} .
\end{aligned}
$$

This shows that we can use a discretization of the diffusion coefficient of the form $a\left(z_{i-1}, z_{i}\right)=$ $\frac{f\left(z_{i-1}\right)+f\left(z_{i}\right)}{2}$ and still have a positivity preserving finite difference scheme as long as we also change the definition of the numerical second derivative. 


\subsection{Computational example}

We now present a computational example, from [103], that illustrates the effectiveness of using a positivity preserving (EDS) scheme over a generic one. Consider the example discussed earlier with initial condition (10) and $n=1 / 2$. We discussed the formation of a finite time singularity that exhibited a discontinuiuty in third derivative as $h \rightarrow 0$. From $[13,11,24]$ we know that the solution can be continued in time as the limit of a sequence of positive approximations. That is, the regularization

$$
h_{\epsilon t}+\left(f_{\epsilon}\left(h_{\epsilon}\right) h_{\epsilon x x x}\right)_{x}=0, f_{\epsilon}\left(h_{\epsilon}\right)=\frac{h_{\epsilon}^{4} f\left(h_{\epsilon}\right)}{\epsilon f\left(h_{\epsilon}\right)+h_{\epsilon}^{4}} .
$$

Since $f_{\epsilon}\left(h_{\epsilon}\right) \sim \frac{h_{\epsilon}^{4}}{\epsilon}$ as $h_{\epsilon} \rightarrow 0$ we know that for all $\epsilon>0$ the analytical solution of the regularized problem is positive. In [18], a nonnegative weak solution is computed numerically by taking successively smaller values of $\epsilon$ in (43). That paper used a scheme of the type (16) with $a\left(s_{1}, s_{2}\right)=f\left(0.5\left(s_{1}+s_{2}\right)\right)$. A fine grid is required in order to resolve the spatial structure and keep the numerical solution positive in order to continue the computation. Here we show that an entropy dissipating scheme does a much better job at computing this problem without requiring excessive spatial resolution.

\section{COARSE GRID COMPUTATION. GENERIC SCHEME.}

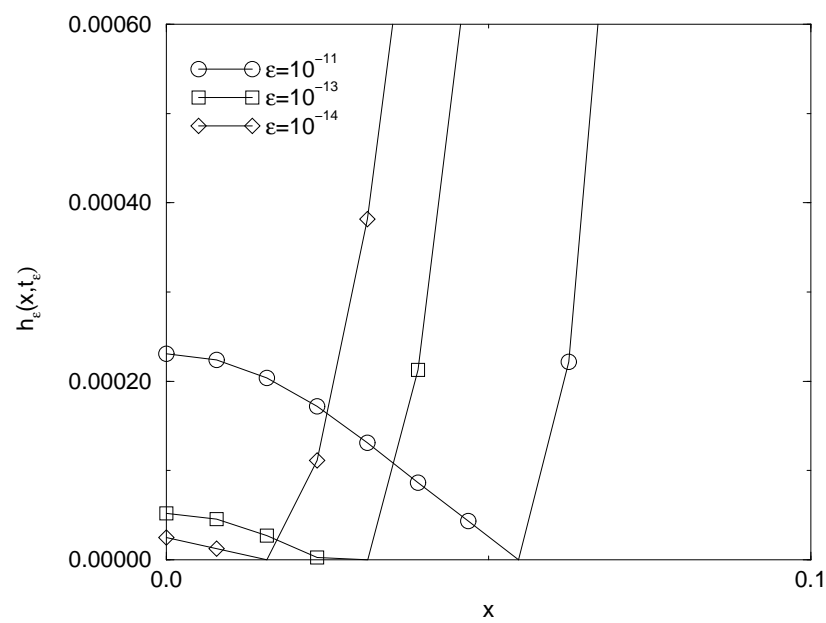

Figure 3: Failed attempt to compute, using a generic difference scheme on a coarse grid, the positive approximations of the weak continuation after the initial singularity described in Figure 2. Final times $t \approx 0.00086,0.00076$ and 0.00074 correspond to $\epsilon=10^{-11}, 10^{-13}$ and $10^{-14} .128$ grid points on $[0,1] \cdot \log _{10}(\min \Delta t)=-14$. We could not continue computing beyond these times since the numerical solution becomes negative.

Figure 3 shows the computational results obtained by the generic scheme for three values of the regularization parameter $\epsilon=10^{-11}, 10^{-13}$ and $10^{-14}$ with uniform grid of 128 points 
on $[0,1]$. In all runs we attempted to reach a final time of $t=10^{-3}$. However, the generic scheme developed a singularity earlier, which made us unable to compute the solution at the time prescribed. Figure 4 shows the results of the entropy dissipating scheme for the same input. In this case we successfully computed the numerical solution up to $t=10^{-3}$. Note that in both cases we used the same purely implicit method for time integration choosing the time step $\Delta t$ small enough to ensure that the discrete time system shows the same behavior as a continuous time one. Figure 5 shows the results obtained by the entropy dissipating

\section{COARSE GRID COMPUTATION. ENTROPY DISSIPATING SCHEME.}

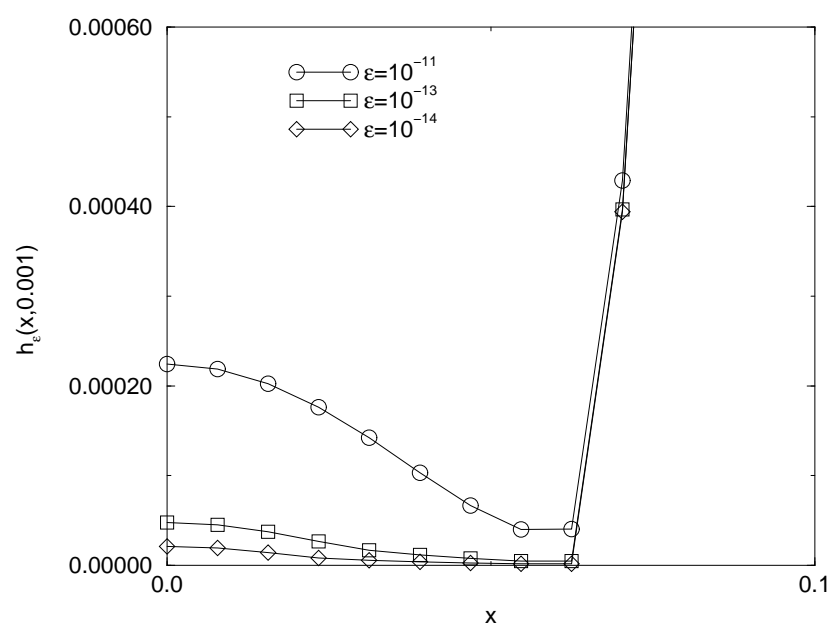

Figure 4: Successful attempt to compute, using an entropy dissipating scheme on a course grid, the numerical solution at fixed time $t=0.001, \epsilon=10^{-11}, 10^{-13}$, and $10^{-14}$. 128 grid points on $[0,1] . \log _{10}(\min \Delta t)=-6.6$ for $\epsilon=10^{-11},-7.2$ for $\epsilon=10^{-13}$ and -7.4 for $\epsilon=10^{-14}$.

scheme on a much finer grid, a uniform grid of 1024 points on [0,1]. Note that even though the graphs look much smoother now, they show very good agreement with those shown in Figure 4.

\section{Driven films and undercompressive shocks}

In many industrial and biological applications thin films are driven by external body forces such as gravity $[60,92]$ or centrifugal forces $[48,71]$. In other words, the external forces instill a convective flow within the film which causes it to spread across a substrate. Under these driving forces the front of the spreading film can become unstable and finger. Moreover, surface stresses, such as that produced by a thermal gradient, can produce very similar fingering patterns [35]. A common feature in these works is the observation that the base state before the instability has, near the contact line, a thick "bump" (frequently termed a capillary ridge) that is responsible for the linear instability.

Some recent driven film experiments, however, studying the problem of a thin film driven by a thermal gradient with an opposing gravitational force have yielded some surprising 


\section{FINE GRID COMPUTATION. ENTROPY DISSIPATING SCHEME.}

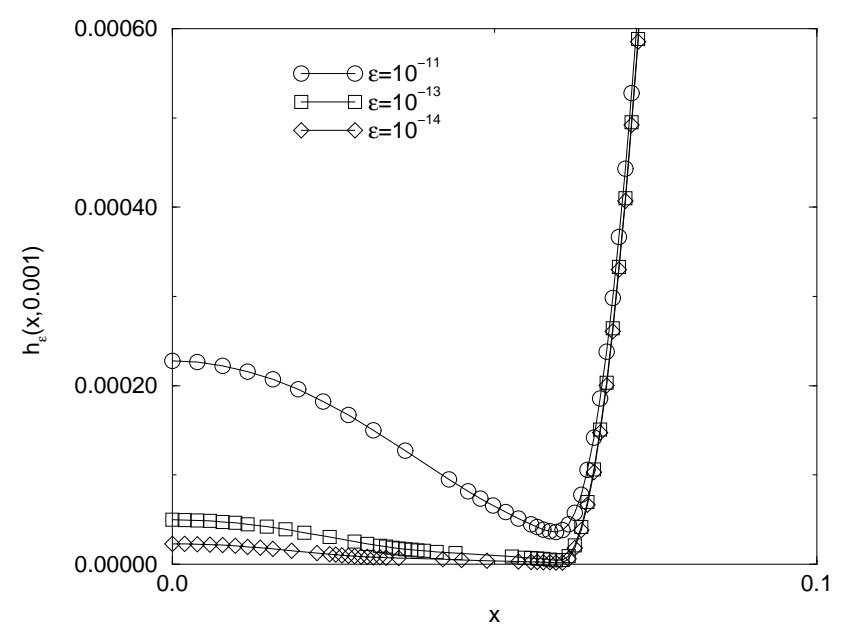

Figure 5: Comparison of computation in Figure 4 with numerical solution on a fine grid. Same fixed time $t=0.001, \epsilon=10^{-11}, \epsilon=10^{-13}$ and $\epsilon=10^{-14} .1024$ grid points on $[0,1]$. $\log _{10}(\min \Delta t)=-6.7$ for $\epsilon=10^{-11},-7.0$ for $\epsilon=10^{-13}$ and -7.3 for $\epsilon=10^{-14}$.

results. It was noticed that the front developed what appeared to be a pronounced capillary ridge (sometimes 2.5 times the thickness of the bulk film) with no apparent fingering.

In this section we consider this problem using a combination of analysis and two-dimensional numerical simulations. There are strong similarities between this problem and many other driven flow problems and the majority of the analysis contained herein is easily adaptable to their study.

\subsection{An experiment}

We have in mind a particular experimental problem, in which both surface stresses and body forces are present, leading to two different kinds of convective motion. However many of the ideas presented here apply to simpler geometries.

We consider an experiment designed and studied by Cazabat's group in Paris [20, 34, 35, $84,83]$. A liquid film is driven from a macroscopic reservoir onto a tilted plate by a surface tension gradient, induced by a constant surface temperature gradient externally imposed along the plate. The liquid is a nonvolatile polydimethylsiloxane (PDMS) which completely wets the substrate, an oxidized silicon wafer. The experiments are prepared with a 7- $\AA$-thick monolayer of PDMS on the surface. Note that this sets a height scale for the precursor layer in the model of the next section.

To increase the film thickness the plate is tilted at an angle from the vertical. Capillarity (and therefore the curvature of the meniscus between the reservoir and the film) govern the film thickness in this range, allowing for control of the film by a geometrical parameter [46], as compared to increasing the thermal gradient, which would ultimately lead to nonlinear behavior of the fluid. The experimental results are quite unexpected: with increased thickness of the flat part of the film the bump becomes more pronounced, but the contact line becomes 
stable. As we discuss in more detail below, the stable "bump" is actually the combination of two shocks, the leading one being undercompressive and the trailing one being compressive.

\subsection{The mathematical model}

We consider a mathematical model based on a lubrication approximation (see $[21,20]$ and references therein):

$$
\frac{\partial u}{\partial t}+\frac{\partial}{\partial x}\left(\frac{\tau u^{2}}{2 \eta}-\frac{\rho g u^{3} \sin \alpha}{3 \eta}\right)=-\nabla \cdot\left[\left(\frac{\gamma u^{3} \nabla \Delta u}{3 \eta}\right)+\left(\frac{\rho g u^{3} \cos \alpha}{3 \eta} \nabla u\right)\right],
$$

where the film height $u$ is a function of position along the incline $x$ and of the transverse variable $y$ as well as of time, $t$. The constants $\tau, \eta, \rho, g, \gamma$ and $\alpha$ respectively represent the temperature gradient, viscosity, density, gravity, surface tension and angle of inclination from the horizontal.

The left hand side of (44) resembles a scalar hyperbolic equation featuring a non-convex flux in the $x$-direction; we discuss hyperbolic conservation laws in detail below. The first term in the flux corresponds to flow driven by the Marangoni stress, while the second represents flow driven by the component of gravity tangential to the substrate. This is coupled to the second and fourth order diffusive regularizations on the right hand side. Physically, the second order term represents the effects of gravity normal to the substrate whereas the fourth order diffusive term arises from surface tension attempting to reduce gradients in the film profile.

Typically, a second order diffusive regularization on its own restricts the existence of shocks to those of purely compressive form. However, the additional fourth order regularization provides an opportunity for shocks of undercompressive form to also appear in the solution of (44). We discuss these details further in terms of a phase space analysis below.

Application of the dimensional rescaling

$$
u=H \hat{u}, \quad x=l \hat{x}, \quad t=T \hat{t}
$$

to (44) yields the relevant scales

$$
H=\frac{3 \tau}{2 \rho g \sin \alpha}, \quad l=\left(\frac{3 \gamma \tau}{2 \rho^{2} g^{2} \sin ^{2} \alpha}\right)^{1 / 3}, \quad T=\frac{2 \eta}{\tau^{2}}\left(\frac{4}{9} \tau \gamma \rho g \sin \alpha\right)^{1 / 3} .
$$

We arrive at the non-dimensional problem (the hats have been dropped for ease of notation)

$$
\frac{\partial u}{\partial t}+\frac{\partial}{\partial x}[F(u)]=-\nabla \cdot\left(u^{3} \nabla \Delta u\right)+D \nabla \cdot\left(u^{3} \nabla u\right)
$$

where

$$
D=\left(\frac{9 \tau^{2}}{4 \gamma \rho g}\right)^{1 / 3} \frac{\cot \alpha}{(\sin \alpha)^{1 / 3}}
$$

and

$$
F(u)=u^{2}-u^{3}
$$


For much of the experimental work done to date, $D \ll 1$, and thus we we focus on the reduced equation

$$
\frac{\partial u}{\partial t}+\frac{\partial}{\partial x}[F(u)]=-\nabla \cdot\left(u^{3} \nabla \Delta u\right)
$$

A detailed investigation of the role of the second order diffusive term in (47) in terms of the size of $D$ can be found in [74]. We also consider the problem in a reference frame moving with speed $s$ and so for the rest of this section we adjust $f(u)$ from (49) to be

$$
F(u)=u^{2}-u^{3}-s u .
$$

To relieve the no-slip paradox that occurs at a moving contact line (as discussed in Section 3.1), we assume the presence of a flat precursor film of thickness $u_{+}$ahead of the film (this being consistent with the precursor layer discussed in the experimental details). Behind the advancing front is a flat film with thickness $u_{-}$controlled by the meniscus [46]. Thus the equations (50)-(51) are coupled to the far-field conditions

$$
u \rightarrow u_{-} \text {as } x \rightarrow-\infty, \quad u \rightarrow u_{+} \text {as } x \rightarrow+\infty .
$$

We note that equation (50) is nearly identical in form to (5), the only difference being the addition of the convective term due to the external driving mechanisms. It is this term that allows shocks or jump discontinuities to form in the solution of (50).

It is useful here to review the role of conservation laws in general. A more comprehensive discussion can be found in [70]. Conservation laws are first order, hyperbolic equations of the form

$$
u_{t}+[F(u)]_{x}=g(u, t, x)
$$

where the equation is called homogeneous if $g \equiv 0$. When the flux $\mathrm{F}(\mathrm{u})$ is a nonlinear function of $\mathrm{u}$ it is well known that shocks may develop from smooth initial data. Before the shock forms the solution is classical and the method of characteristic gives the unique solution. After the shock forms, the solution is no longer classical, but still makes sense as a weak solution involving integration of the PDE against smooth test functions. This machinery allows us to interpret spatial jumps in the solution of a first order equation and yields a relationship, called the Rankine-Hugoniot jump condition, between the shock speed and the left and right states of the shock. Where it is smooth, the weak solution corresponds to a classical solution. Uniqueness of the weak solution requires an additional condition that arises from the physics of the problem. For (53), the admissible solution is taken to be the one that satisfies the Lax entropy condition, namely that

$$
F\left(u_{-}\right)>s>F\left(u_{+}\right),
$$

where $\mathrm{s}$ is the shock speed and the subscripts on $\mathrm{u}$ represent the left and right states on either side of the shock, consistent with (52). The admissible solution is sometimes termed a Lax shock and is also called compressive due to its form in characteristic space. The Lax entropy condition, however, is only a local condition and for a globally admissible solution we must also satisfy the Oleinik chord condition (the Lax entropy condition can be shown to arise out of a simple perturbation analysis of the Oleinik chord condition). We note that 
for fluxes with at most one inflection point, the Lax entropy condition alone is sufficient to prove admissibility, but this is not true in general. The Oleinik chord condition, which states that on the graph of the flux function, the chord connecting a left and right state of a shock can not cross the flux function, is the correct condition for more general fluxes. One can observe this to be the case by analyzing the limiting traveling wave solution of the viscously regularized conservation law

$$
u_{t}+[F(u)]_{x}=\varepsilon \Delta u \quad \text { as } \quad \varepsilon \rightarrow 0 .
$$

It turns out that the choice of a second order regularization is rather subtle and taking a higher order regularization leads to some surprising alternatives. It has recently been shown that problems of the form

$$
u_{t}+(F(u))_{x}=-\nabla \cdot\left(u^{n} \nabla \Delta u\right)
$$

allow undercompressive shocks to form and we must reformulate our notion of admissibility. Undercompressive and other non-classical shocks have been well studied in systems of conservation laws $[1,31,61,89,90,100]$. For the scalar law case, they were thought to be a purely mathematical curiosity and were not believed to have any experimental basis. The experiment we discuss here is perhaps the first example of a physical undercompressive shock for a scalar law problem. Of particular interest is the stability of these undercompressive solutions and their numerical modeling. Numerical schemes are typically designed to seek out compressive shocks and ignore any undercompressive effects. The creation of codes which capture the undercompressive behavior has therefore become an area of growing interest. Additionally, in numerical simulations and physical experiments it is found that the creation of an undercompressive shock is typically coupled with the appearance of a standard compressive shock and it is important to know how these multiple shock structures are effected by small perturbations.

We now return to the problem at hand. For each value of $u_{+}$(i.e. a particular precursor thickness) there are a number of values of $u_{-}$for which transitions/bifurcations in the solution structure occur. We assume that $u_{+} \ll 1$ (for our purposes, the analysis for any $u_{+}<1 / 3$ is equivalent; the second derivative of the flux function changes sign at $u_{+}=1 / 3$ and it is impossible to generate compressive shocks with $u_{-}>u_{+}$when $u_{+}>1 / 3$ ).

We begin by reviewing the possible scenarios for long time behavior of the one dimensional problem:

(I) For $u_{+}<u_{-}<u_{1}\left(u_{1}\right.$ dependent on $\left.u_{+}\right)$there exists a unique capillary Lax shock connecting $u_{-}$to $u_{+}$.

(II) For $u_{1}<u_{-}<u_{2}\left(u_{2}\right.$ dependent on $\left.u_{+}\right)$there are multiple capillary Lax shock profiles connecting $u_{-}$to $u_{+}$. There is a critical value of $u_{-}$, denoted here by $u_{*}$ for which an infinite number of capillary Lax shocks exist.

(III) For $u_{-}>u_{2}$ there are no capillary Lax shocks connecting $u_{-}$to $u_{+}$. There is, however, a critical value of $u_{-}=u_{U C}$ (dependent on $u_{+}$) for which a single undercompressive capillary shock exists.

For a single shock profile (compressive or undercompressive) the unperturbed base state is given by the one-dimensional traveling wave solution of the evolution equation (50) [21] (see also $[60,64]$ for comparison). Imposing the solution $u=u(x)$ (the traveling wave solution becomes stationary in the moving reference frame if $s$ is chosen suitably in (51), cf. (59)) yields 


$$
[F(u)]_{x}+\left(u^{3} u_{x x x}\right)_{x}=0
$$

Equation (57) is immediately integrable, the constant of integration being specified by the far-field conditions (52). Application of these conditions yields

$$
\begin{aligned}
& F\left(u_{+}\right)=u_{+}^{2}-u_{+}^{3}-s u_{+}=k, \\
& F\left(u_{-}\right)=u_{-}^{2}-u_{-}^{3}-s u_{-}=k,
\end{aligned}
$$

which can be rearranged to give the Rankine-Hugoniot condition for the speed of the shock

$$
s=\frac{u_{+}^{2}-u_{+}^{3}-\left(u_{-}^{2}-u_{-}^{3}\right)}{\left(u_{+}-u_{-}\right)} .
$$

We choose this value of $s$ in (51) for both numerical and analytical convenience.

\subsection{Traveling waves with fourth order diffusion.}

The admissibility of non-classical shocks with higher order diffusion is related to the admissibility of traveling waves for this problem [21]. These solutions satisfy the third order ordinary differential equation (cf. (57))

$$
F(u)-F\left(u_{+}\right)=-u^{3} u^{\prime \prime \prime}
$$

(In integrating the equation once, we have assumed $u^{\prime \prime \prime}(x) \rightarrow 0$ and $u(x) \rightarrow u_{+}$as $x \rightarrow \infty$ ). Equation (60) has two parameters $u_{+}$and $s$, which appear in $F$ in (51). The traveling reference frame with $s$ appearing in (51) results in stationary waves.

Writing (60) as

$$
u^{\prime \prime \prime}=g\left(u ; u_{+}, s\right)
$$

where

$$
g\left(u ; u_{+}, s\right)=-u^{-3}\left(F(u)-F\left(u_{+}\right)\right),
$$

we see that the equilibrium $u=u_{-}$(zeroes of $g\left(. ; u_{+}, s\right)$ ) is given by the Rankine-Hugoniot condition (59) for shocks. Moreover, the linearized ordinary differential equation $\tilde{u}^{\prime \prime \prime}=$ $\frac{\partial g}{\partial u}\left(u_{-} ; u_{+}, s\right) \tilde{u}$ has three eigenvalues, namely the three cube roots of $\frac{\partial g}{\partial u}\left(u_{-} ; u_{+}, s\right)$. Note further that $\frac{\partial g}{\partial u}\left(u_{-} ; u_{+}, s\right)=-\frac{1}{u_{-}^{3}}\left(F^{\prime}\left(u_{-}\right)\right)$, so that the sign of $\frac{\partial g}{\partial u}\left(u_{-} ; u_{+}, s\right)$ is related to whether characteristics at $u_{-}$, traveling with speed $F^{\prime}\left(u_{-}\right)$, are faster or slower than the speed $s$ of the traveling wave.

To further understand equilibria, and trajectories joining different equilibria, we write equation (61) as a first order system:

$$
\begin{aligned}
u^{\prime} & =v, \\
v^{\prime} & =w, \\
w^{\prime} & =g\left(u ; u_{+}, s\right) .
\end{aligned}
$$


For the specific equilibrium $(u, v, w)=\left(u_{-}, 0,0\right)$, with $g\left(u_{-} ; u_{+}, s\right)=0$, the three cube roots of $\frac{\partial g}{\partial u}\left(u_{-} ; u_{+}, s\right)$ give rise to the following structure of invariant manifolds containing the equilibrium.

(i) If $F^{\prime}(u)<s$, then $\frac{\partial g}{\partial u}\left(u ; u_{+}, s\right)>0$, so that $(u, 0,0)$ has a one-dimensional unstable manifold and a two dimensional stable manifold on which solutions spiral into the equilibrium due to the complex conjugate pair of eigenvalues with negative real part.

(ii) If $F^{\prime}(u)>s$, then $\frac{\partial g}{\partial u}\left(u ; u_{+}, s\right)<0$, so that $(u, 0,0)$ has a one-dimensional stable manifold and a two dimensional unstable manifold on which solutions spiral away from the equilibrium due to the complex conjugate pair of eigenvalues with positive real part.

Now equilibria of (63) correspond to intersections of the line with zero slope through $\left(u_{+}, F\left(u_{+}\right)\right)$with the graph of $F$. Alternatively, we can fix $u_{-} \neq u_{+}$and define $s$ through the Rankine-Hugoniot condition (59). Specifically, for the flux function $F(u)=u^{2}-u^{3}-s u$,

$$
s=u_{-}+u_{+}-u_{-}^{2}-u_{-} u_{+}-u_{+}^{2} .
$$

We now consider the case of a weak Lax shock, specifically $0<u_{+}<u_{-}<1 / 3$, with $u_{-}-u_{+}$small, in detail; a similar analysis holds for the other cases. Then $\left(u_{-}, 0,0\right)$ has a two-dimensional unstable manifold and $\left(u_{+}, 0,0\right)$ has a two-dimensional stable manifold. If they intersect transversally (away from the equilibria), then the curve of intersection is a trajectory for $(63)$ from $\left(u_{-}, 0,0\right)$ to $\left(u_{+}, 0,0\right)$. We refer to the corresponding solution of the partial differential equation (50) as a capillary shock profile from $u_{-}$to $u_{+}$. The existence of capillary shock profiles for weak Lax shocks away from the inflection point $u=1 / 3$ is provided by the analysis of Kopell and Howard [67].

\subsection{Structure of phase space}

We next investigate the appearance of capillary shock profiles for different values of $u_{-}$, and hence different $s$, by numerically exploring the structure of the phase space. In order to do so, we determine the unstable and stable manifolds of the three equilibria

$$
B=\left(u_{+}, 0,0\right), \quad M=\left(u_{-}, 0,0\right), \quad \text { and } \quad T=\left(1-u_{-}-u_{+}, 0,0\right),
$$

(with $u_{+}<u_{-}<1-u_{-}-u_{+}$).

Certain trajectories are visualized in a perspective plot of the three-dimensional phase space (see Figure 6(a) for $u_{-}=0.33$ ). Additionally, we show the intersection of the stable manifold $W^{s}(B)$ of $B$, the unstable manifold $W^{u}(M)$ of $M$, and the unstable manifold $W^{u}(T)$ of $T$ with the plane

$$
P=\left\{\left(u, u^{\prime}, u^{\prime \prime}\right) ; u=\left(2 u_{-}+u_{+}\right) / 3\right\}
$$

in Figure 6(b). We refer to the plane $P$ as a Poincaré section. Since $W^{s}(B)$ and $W^{u}(M)$ are surfaces, their intersections with $P$ appear as two curves, whereas the one-dimensional manifold $W^{u}(T)$ appears in the Poincaré section as a point. Capillary profiles for Lax shocks correspond to intersections of $W^{u}(M)$ and $W^{s}(B)$. A capillary profile for an undercompressive shock appears when $W^{s}(B)$ contains the curve $W^{u}(T)$.

As an example of the use of Poincaré sections in the study of multi-dimensional differential equations we study the case II from Section 3.2, $u_{1}<u_{-}<u_{2}$, corresponding to multiple capillary shock profiles; a full description of the entire phase space can be found in [21]. 
For each value of $u_{+}<1 / 3$ there is a range of values for $u_{-}>u_{1}$, for which there exists more than one orbit connecting $M$ to $B$. This range of $u_{-}$extends up to a finite upper bound $u_{2}$, For $u_{+}=0.1$, we find numerically

$$
u_{2}=0.3479
$$

The number of connections can be computed numerically by examining the number of intersection points of the curves $W^{u}(M) \cap P$ and $W^{s}(B) \cap P$ on the Poincaré section.

For $u_{-}=0.33$, the Poincaré section, shown in Figure 6(b), indicates three intersections of $W^{s}(B) \cap P$ and $W^{u}(M) \cap P$. Figure 6 (a) shows the corresponding orbits in three dimensional phase space; on the scale of the graph, two of the orbits nearly coincide with the largest separation near $T$.

There is a special value of $u_{-}$, which we denote by $u_{*}$, for which $W^{U}(T)$ intersects $W^{S}(B)$. The existence of this connection was proved rigorously by the first author and Shearer in [23]. At this special value, we observe in the phase portrait, infinitely many turns in $W^{u}(M) \cap P$, and consequently a countably infinite number of intersections of $W^{u}(M) \cap P$ with $W^{s}(B) \cap P$.To understand this, we first remark that, in the neighborhood of $T$, the connection from $M$ to $T$ is governed by the two complex eigenvalues of the linearization of (63) around this equilibrium. These eigenvalues have negative real part, so that the trajectory approaches $T$ through a spiral with an infinite number of turns. A neighboring trajectory, contained in $W^{u}(M)$ but not in $W^{s}(T)$, will initially stay close to the orbit connecting $M$ to $T$, and undergo some turns, until it finally is repulsed from the vicinity of $T$ along one of the two branches of $W^{u}(T)$. The infinite number of intersections of $W^{u}(M) \cap P$ with $W^{s}(B) \cap P$ when $u=u_{*}$ corresponds to an infinite number of different orbits connecting $M$ to $B$. Each orbit corresponds to a different traveling wave solution of (50).

Every solution connects the same two far-field constant states and therefore (by the Rankine-Hugoniot condition (59)) has the same speed. Note that Figure 6(b) resolves only a few turns of the spirals of $W^{u}(M) \cap P$. In numerical trials we could resolve a total of six turns and five intersection points, in $W^{u}(M) \cap W^{s}(B) \cap P$. The accumulation point of the intersection points in the Poincaré section corresponds to the special orbit connecting $T$ to $B$. This orbit yields a special traveling wave solution of (50). This is an undercompressive capillary shock. Note also that $u_{*}$ and $u_{U C}$ are related by $u_{U C}=1-u_{+}-u_{*}$.

The analysis of traveling waves can be carried out for the more general problem resulting from (47) with $D>0$. In [23] it was rigorously proved that for any $u_{+}<1 / 3$ and $D$ sufficiently small there exists a special shock speed $s^{*}$ for which a heteroclinic orbit from $T$ to $B$ exists. This orbit corresponds to an undercompressive traveling wave connecting $u_{+}$to $u_{U C}$. However, for $D$ large enough, no such connection exists and undercompressive shocks cannot arise in the solution. The phase space computations in [21] were extended to the case of positive $D$ in (48) by Münch [74] and to the case of traveling waves satisfying the Navier slip condition (in lieu of having a precursor film, so that there is a true contact line) in [32]. The full problem with van der Waals interactions (wetting as as opposed to dewetting, discussed in the next section) was recently considered by Golovin et al [51].

\subsection{Two-dimensional numerical simulations}

When the fingering instability arises the dynamics are no longer one-dimensional and we must study the full two-dimensional numerics to understand the phenomena. We solve (50) 


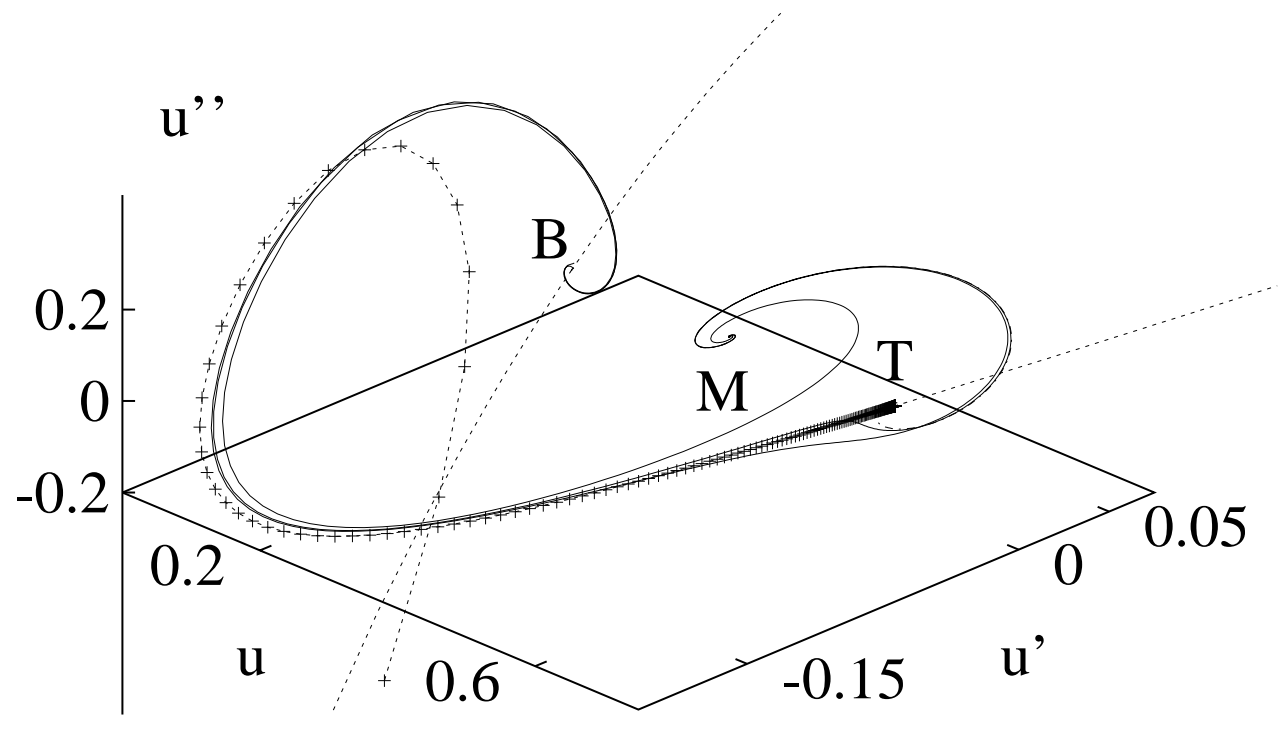

(a) Perspective view

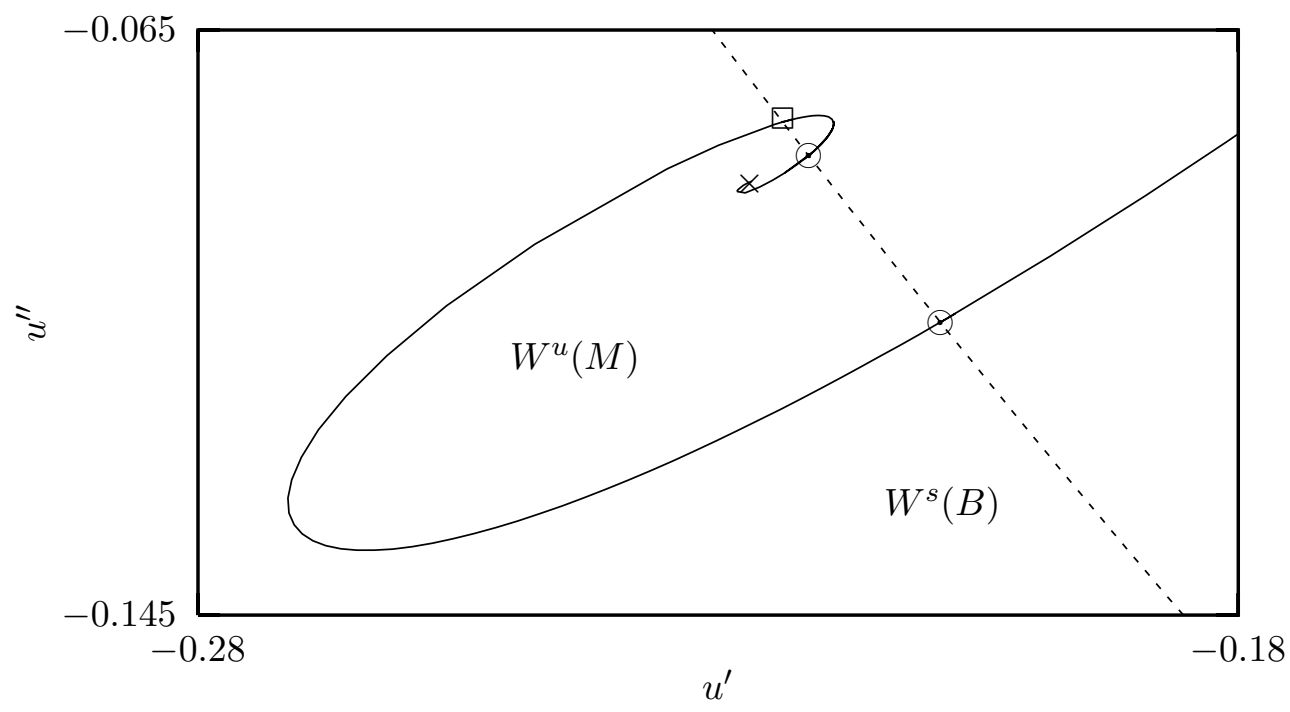

(b) Poincaré section $P$

Figure 6: Phase portrait for $u_{-}=0.33$ : Solid lines in (a) represent the three connections from $M$ to $B$, which appear as three intersection points $W^{u}(M) \cap W^{s}(B) \cap P$ in (b). Circles and boxes indicate whether they correspond to stable or unstable traveling wave solutions of the PDE (50), respectively. For better visibility, one branch of $W^{u}(T)$ has been emphasized with ' + '. We note that this diagram is plotted with the parameter $s$ set to zero in (51). 
using the ADI scheme described below. Such methods have a long history of application to second order and linear fourth order problems. More recently they have also been used in other driven thin film problems (see, for example, $[86,42]$ ). The method applied here is an extension of those investigated in [10].

There is an inherent fear in using an ADI scheme for higher order parabolic problems as there are no convergence or existence results available. However, as illustrated in [10] schemes for fourth order problems appear to perform equally as well as other possible schemes and have a distinct speed advantage requiring only $O(N)$ computations at each time step. It is also noteworthy that starting from one-dimensional initial conditions (i.e. with no variation in the transverse $y$ direction) our scheme computes solutions with cross-section that matches the one-dimensional solutions found in [21]. We feel these comments justify the use of the method here.

It has been previously shown in [98] that it is impossible to attain $O\left((\Delta t)^{2}\right)$ accuracy with a two level ADI scheme. Equation (50) is therefore approximated by a three level scheme which is formally $O\left((\Delta t)^{2}\right)$ accurate. We take

$$
\begin{gathered}
\mathcal{L}_{x} u^{*}=\frac{1}{\gamma}\left[\alpha u^{n}+\beta u^{n-1}\right]-\frac{1}{\gamma} \Delta t_{1}\left[\partial_{x}\left(\tilde{f}^{n+1} \partial_{y y x}\right)+\partial_{y}\left(\tilde{f}^{n+1} \partial_{x x y}\right)\right] \tilde{u}^{n+1} \\
-\frac{1}{\gamma} \Delta t_{1} \mathcal{D}_{y} \tilde{u}^{n+1} \\
\mathcal{L}_{y} u^{n+1}=u^{*}+\frac{1}{\gamma} \Delta t_{1} \mathcal{D}_{y} \tilde{u}^{n+1}
\end{gathered}
$$

where

$$
\begin{aligned}
& \mathcal{L}_{x}=\mathcal{I}+\frac{1}{\gamma} \Delta t_{1} \mathcal{P}_{x}+\frac{1}{\gamma} \Delta t_{1} \mathcal{D}_{x}, \quad \mathcal{L}_{y}=\mathcal{I}+\frac{1}{\gamma} \Delta t_{1} \mathcal{D}_{y} \\
& \mathcal{D}_{x}=\partial_{x}\left(\tilde{f}^{n+1} \partial_{x x x}\right), \quad \mathcal{D}_{y}=\partial_{y}\left(\tilde{f}^{n+1} \partial_{y y y}\right), \quad \mathcal{P}_{x} u^{*}=\partial_{x}\left(u^{* 2}-u^{* 3}-U u^{*}\right), \\
& \tilde{u}^{n+1}=\sigma u^{n}+\rho u^{n-1}, \quad \tilde{f}^{n+1}=f\left(\tilde{u}^{n+1}\right)
\end{aligned}
$$

and the constants $\alpha, \beta, \gamma, \rho$ and $\sigma$ are defined as

$$
\begin{aligned}
& \alpha=\frac{\Delta t_{1}+\Delta t_{2}}{\Delta t_{2}}, \quad \beta=-\frac{\left(\Delta t_{1}\right)^{2}}{\Delta t_{2}\left(\Delta t_{1}+\Delta t_{2}\right)}, \quad \gamma=\frac{\Delta t_{2}+2 \Delta t_{1}}{\Delta t_{2}+\Delta t_{1}}, \\
& \rho=-\frac{\Delta t_{1}}{\Delta t_{2}}, \quad \sigma=\frac{\Delta t_{1}+\Delta t_{2}}{\Delta t_{2}} .
\end{aligned}
$$

Here $u^{*}$ denotes an intermediate value for the numerical scheme. Note that the three time levels are contained within the definition of $\tilde{u}^{n+1}$ (and therefore $\tilde{f}^{n+1}$ ) which is a linear approximation for $u^{n+1}$ while the effects of including an adaptive time step are seen in the choice of $\alpha, \beta, \gamma, \rho$ and $\sigma$. Most importantly, by using the $\tilde{f}^{n+1}$ approximation for $f^{n+1}$, equation (66) is linear and the only nonlinearity in (65) comes from $\mathcal{P}_{x}$. Although we have made a further approximation to gain linearity in one of the equations this will not introduce errors larger than $O\left((\Delta t)^{2}\right)$ at any time step and we do not believe that the accumulated error 
over several time steps will be any larger than that already introduced by our approximation of (50) by (65)-(66). If this did become an issue, it is a relatively simple matter to introduce an iteration procedure at each time step that updates our approximation $\tilde{f}^{n+1}$ until the difference between successive approximations is below a certain preset tolerance (implying that we are close to the true value of $f^{n+1}$ ); see [10] for a discussion of iteration methodology for these problems.

We now turn our consideration to the choice of suitable discretizations for the spatial derivatives in (65)-(66), including those inherent in $\mathcal{D}_{x}, \mathcal{D}_{y}$ and $\mathcal{P}_{x}$. The discretizations adopted here are cell centered, second order accurate, thereby making the whole scheme $O\left((\Delta t)^{2}+(\Delta x)^{2}\right)$ accurate. The first order derivatives with respect to $x$ are given by

$$
\partial_{x} p_{i, j}=\frac{p_{i+1 / 2, j}-p_{i-1 / 2, j}}{\Delta x}+O\left((\Delta x)^{2}\right)
$$

and similarly for $\partial_{y} p_{i, j}$. The cell centered third derivative takes the form

$$
\partial_{x x x} u_{i+1 / 2, j}=\frac{u_{i+2, j}-3 u_{i+1, j}+3 u_{i, j}-u_{i-1, j}}{(\Delta x)^{3}}+O\left((\Delta x)^{2}\right)
$$

and similarly for $\partial_{y y y} u_{i, j+1 / 2}$. The mixed derivatives are also given by cell centered differences, namely

$\partial_{x y y} u_{i+1 / 2, j}=\frac{u_{i+1, j+1}-u_{i, j+1}-2 u_{i+1, j}+2 u_{i, j}+u_{i+1, j-1}-u_{i, j-1}}{\Delta x(\Delta y)^{2}}+O\left((\Delta x)^{2}\right)+O\left((\Delta y)^{2}\right)$

and similarly for $\partial_{y x x} u_{i, j+1 / 2}$.

Finally, we must select a discretization for the nonlinear diffusion in the fourth order term. We take the standard averaged approximation

$$
f_{i+1 / 2, j}^{n+1}=\frac{1}{2}\left(f_{i, j}^{n+1}+f_{i+1, j}^{n+1}\right)+O\left((\Delta x)^{2}\right)
$$

At each time step we solve the nonlinear equation (65) using Newton iteration and then solve the linear equation (66) using a banded matrix solver with LU decomposition. Obviously, the approximations we make here are by no means unique. For a more detailed discussion of ADI methods for high order parabolic problems see [10].

Figure 7 shows a two dimensional simulation of a separating double shock structure. Transverse sinusoidal perturbations are added in front of the leading shock (left). These bumps impact the undercompressive wave (middle) but do not destabilize it. However, their effect results in perturbations traveling along characteristics from the undercompressive wave to the trailing Lax wave (right). In contrast, a perturbation applied to a compressive wave, resulting from single step initial data, produces the characteristic fingering instability shown in Figure 8.

\subsection{Linear stability analysis}

To understand the transition to fingering we must perform a linear stability analysis. We begin by invoking the solution ansatz

$$
u(x, y, t)=\hat{u}(x, t)+\varepsilon h(x, t) \cos (k y) \quad \varepsilon \ll 1 .
$$



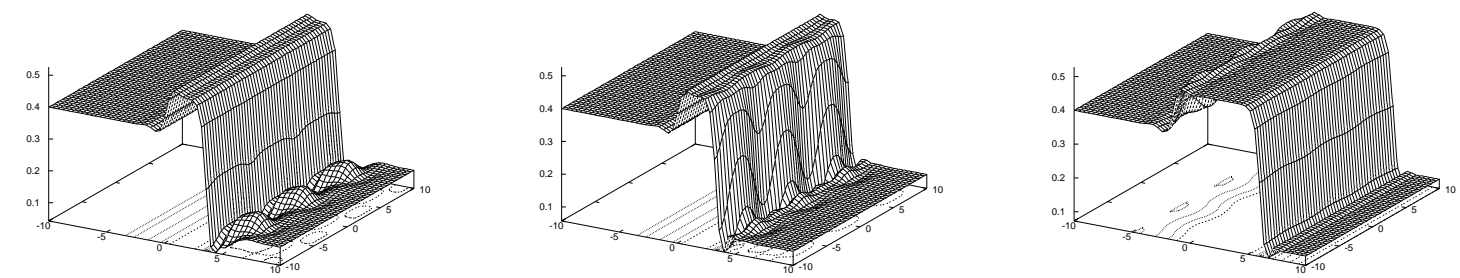

Figure 7: Surface and contour plots showing the effect of transverse perturbations on the double shock structure of an undercompressive-Lax wave pair for the thermal-gravity flux (51).
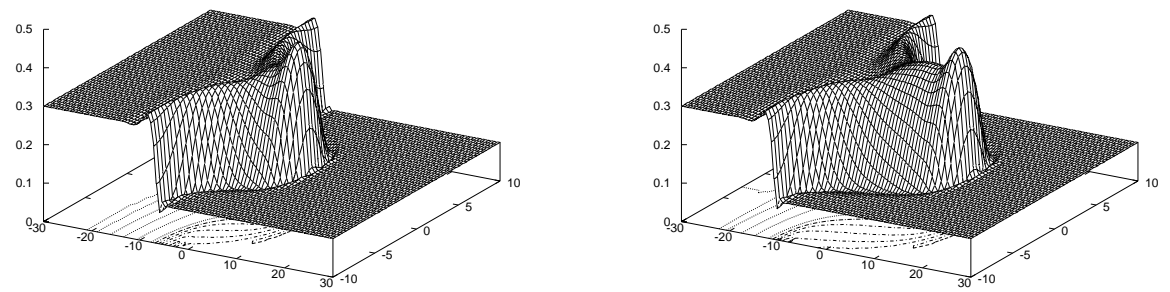

Figure 8: Surface and contour plots showing the fingering instability for a compressive wave. 
and substitution of (67) into (50) leads to a linear evolution equation for $h$ at $O(\epsilon)$.

$$
h_{t}=-\left[h F^{\prime}(\hat{u})\right]_{x}-\left[\partial_{x}\left(\hat{u}^{3} \partial_{x}^{3} h+3 h \hat{u}^{2} \partial_{x}^{3} \hat{u}\right)\right]-k^{2}\left[\partial_{x}\left(\hat{u}^{3} \partial_{x} h\right)+\hat{u}^{3}\left(\partial_{x}^{2} h\right)\right]-k^{4} \hat{u}^{3} h=\mathcal{L}_{k} h
$$

where $k$ denotes the $y$ wavenumber. The symmetry in $y$ leads to only even powers of $k$ in (68).

For self-adjoint problems this linear stability analysis is sufficient to completely describe the behavior of solutions. However, the problem considered here is not self-adjoint and the large time growth rates tell only part of the whole story. There happens to be transient timescales on which growing modes can excite nonlinearities of the original equation [19].

For simplicity of analysis we begin by considering the case of a single, well-established shock (either compressive or undercompressive); in the form of a traveling wave $\hat{u}(x, t)=\hat{u}(x)$. Note that we choose the reference frame to travel at the same speed as the wave, which is equivalent to setting $s$ in (51) so that the speed of the shock is zero. Thus the traveling wave becomes a stationary solution $\hat{u}$. Looking for solutions to (68) of the separable form $h(x, t ; k)=e^{\beta(k) t} \psi(x, k)$ leads to the relationship

$$
\mathcal{L}_{k} \psi=\beta \psi,
$$

so that $\beta$ acts as an eigenvalue with $\psi$ as the corresponding eigenfunction. We are primarily interested in small $k$ corresponding to long wavelength perturbations; large $k$ perturbations are always smoothed rapidly by surface tension effects. In the small $k$ limit we can expand $\beta$ and $\psi$ as power series in even powers of $k$ due to the symmetry in $y$. Whether solutions grow or decay for large time is thereby dependent on the sign of $\beta_{1}$, which can be found from the solvability condition

$$
\beta_{1} \int_{-\infty}^{\infty} \hat{u}_{x} \pi_{0} d x=\int_{-\infty}^{\infty} \pi_{0 x} \hat{u}^{3} \hat{u}_{x x} d x+\int_{-\infty}^{\infty} \pi_{0}\left[F(\hat{u})-F\left(u_{+}\right)\right] d x
$$

where $\pi_{0}(x)$ is the left eigenfunction that satisfies the formal adjoint of $\mathcal{L}_{0}$, namely $\mathcal{L}_{0}^{*} \pi_{0}=0$, where

$$
\mathcal{L}_{0}^{*}=-\frac{d^{3}}{d x^{3}}\left(\hat{u}^{3} \frac{d}{d x}\right)+\left(F^{\prime}(\hat{u})+3 \hat{u}^{2} \frac{d^{3} \hat{u}}{d x^{3}}\right) \frac{d}{d x}
$$

\subsubsection{Compressive shocks}

The work of [17] showed (by application of spectral theory) that for compressive shocks there exists a unique left eigenfunction, $\pi_{0}$, given by the constant function $\pi_{0}=1 /\left(u_{+}-u_{-}\right)$. The fact that $\pi_{0}$ is constant validates the stability analysis considered in [19] and [63]. The solvability condition (70) then simplifies to

$$
\beta_{1}=\frac{1}{u_{+}-u_{-}} \int_{-\infty}^{\infty}\left[F(\hat{u})-F\left(u_{+}\right)\right] d x
$$

where the integral can be calculated numerically once $\hat{u}(x)$ is known. As $u_{+}-u_{-}$is a fixed quantity, the stability of compressive shocks therefore comes down to the sign of the integral in (72) which must change at a critical wavenumber $k_{c}$; for $k<k_{c}$ the compressive shock is unstable whereas for $k>k_{c}$ the shock is stable. 


\subsubsection{Undercompressive shocks}

Analysis of the evolution equation (50) illustrates that double shock structures can form from simple step initial data [21]. In fact, as mentioned previously, we only expect to have an undercompressive shock existing independently for the choice of $u_{-}=u_{U C}$. For $u_{-} \neq u_{U C}$ we must necessarily have either a compressive shock or a undercompressive/compressive shock pair. The double shock structures seen in experiment [20] consist of a leading undercompressive shock followed by a trailing compressive shock. The undercompressive shock is a jump from the precursor film thickness $u_{+}$to a large height $u_{U C}$ while the compressive shock is a jump from $u_{U C}$ down to the flat film height $u_{-}$, which is fixed by the curvature of the meniscus in the experiment.

It was shown in [17] that the unique left eigenfunction for undercompressive shocks is not constant and takes no simple analytic form. A far-field and numerical study led to the understanding that for a range of $k>0$, the right eigenfunction grows exponentially as $x \rightarrow-\infty$ and this is the mechanism by which perturbations are swept away from behind the undercompressive front. The fact that perturbations do not remain localized to an undercompressive shock has important implications for these shock pairs. From the linear stability analysis above, we see that it possible for the perturbation spreading from the undercompressive shock to reach the compressive shock which may then destabilize leading to a fingering instability down the plate toward the fluid bath. This scenario is consistent with the numerical simulation shown in Figure 7. The important consequence of this analysis is that undercompressive shocks are stable to all transverse perturbations; it is these special stability properties which provide the mechanism for producing stable moving contact lines. In contrast, for compressive shocks there exists a band of long wavelength perturbations capable of destabilizing the shock and triggering a fingering instability.

\section{Dewetting films}

We now turn to the problem of dewetting films. The competition of attractive van der Waals forces and short range repulsive forces, like Born repulsion, can produce complex instabilities in layers of thin liquid films on solid substrates. Experimental studies have shown this behavior for polymer films [66, 81, 87, 88, 101], liquid crystal films [58, 93, 94], liquid metals $[29,58]$ and evaporating films $[41,76]$. During these dewetting processes, large droplets are formed which are connected by ultra-thin films. In analogy to spinodal decomposition in phase separation for binary alloys described by the Cahn-Hilliard equation, this evolution is sometimes called spinodal dewetting $[72,73,76]$.

We consider a model and results from [28]. The lubrication approximation for dewetting films under the influence of both attractive van der Waals forces and short range repulsive forces has a general form $[37,78]$

$$
\frac{\partial h}{\partial t}-\nabla \cdot(f(h) \nabla p)=0
$$

where the pressure $p$ in the thin film is given by

$$
p=P(h)-\Delta h
$$


The explicit form of the nonlinear mobility $f$ again depends on the boundary condition at the liquid-solid interface, as in (5). The forms

$$
f(h)=h^{3}, \quad \text { and } \quad f(h)=h^{3}+\beta h^{b},
$$

with $\beta>0, b \in(0,3)$, correspond to no-slip and various classes of slip boundary conditions respectively.

The term $P(h)$ encompasses forces exerted on the film, such as a hydrostatic body force and disjoining/conjoining intermolecular forces due to van der Waals interactions and Born repulsion [43]. For dewetting, we consider functions

$$
P(h)=\frac{1}{h^{n}}-\frac{\epsilon^{m-n}}{h^{m}}=\frac{1}{h^{n}}\left(1-\left[\frac{\epsilon}{h}\right]^{m-n}\right), \quad 0<n<m
$$

where $\epsilon$ is a small positive parameter [42]. The standard 6-12 Lennard-Jones potential [62, 72, $73,78]$ corresponds to the exponents $(n, m)=(3,9)$ in this model. The $h^{-n}$ term describes the aforementioned long-range attractive force, while the second term in (76) models the short range stabilizing effect of Born repulsion. Oron et al. [76, 77] consider (76) with $(n, m)=(3,4)$ to describe a thin film on a layered solid substrate. Our results show that the solutions of these models have the same qualitative structure for a large set of $(n, m)$ values.

Early studies of van der Waals driven instabilities of thin films, by Williams and Davis $[33,96]$ and de Gennes [37], considered this problem without repulsive pressure terms, i.e. $P(u)$ given by equation (76) with $\epsilon=0$. In general, classical solutions of this problem can cease to exist in finite time due to singularities which occur if the film ruptures, $u \rightarrow 0$. In this context, for $n=3$, self-similar finite-time rupture solutions were studied by Zhang and Lister [102] and Witelski and Bernoff [99, 97].

In contrast, for $\epsilon>0$, the problem is globally well-posed, and solutions exist for all times $[28,54,55,56]$. Numerical simulations of thin films with generalized van der Waals disjoining pressure show complex pattern formation during which films evolve to a meta-stable state composed of a collection of droplets connected by a thin film of thickness approximately $\epsilon$. A comparison with physical experiments shows striking similarities to the characteristic features of dewetting including the formation of capillary ridges at the edges of growing holes (cf. [56] and [85] for drying dewetting paint).

We show that the parameter $\epsilon$ in (76) gives a minimum thickness for non-trivial thin film equilibrium states. Viewed as a regularization, it is natural to consider the limit of these 'post-rupture' solutions as $\epsilon \rightarrow 0$. We show via numerical simulations and asymptotic analysis of the equilibria, that concentrations [44] occur in this model. That is, this limit makes sense only as a measure; for fixed times after rupture numerical simulations show the $\epsilon \rightarrow 0$ limit concentrates mass as a $\delta$-distribution.

Appropriate boundary conditions for equation (73) corresponding to the physical problem of a fluid confined in an impermeable solid container are

$$
\mathbf{n} \cdot \nabla p=0, \quad \mathbf{n} \cdot \nabla h=0, \quad \text { on } \quad \partial \Omega .
$$

The latter condition specifies a $90^{\circ}$ contact angle between the fluid surface and vertical boundaries at $\partial \Omega$. This condition makes a uniform fluid layer, $h(x, y)=\bar{h}$, an exact solution of the full problem and is physically equivalent to neglecting any positive or negative meniscus 
due to wetting or non-wetting properties of the container holding the fluid. By the former condition, mass is conserved, as is the average film thickness $\bar{h}$ which is given by

$$
\bar{h}=\frac{1}{\mathcal{A}} \int_{\Omega} h d A,
$$

where $\mathcal{A}=\operatorname{area}(\Omega)$. We use $\bar{h}$ as a bifurcation parameter for the set of equilibrium solutions of (73). An energy, or Liapunov integral, for (73) is given by

$$
\mathcal{E}=\int_{\Omega} \frac{1}{2}|\nabla h|^{2}+Q(h) d A,
$$

where $Q$ is the primitive of $P$,

$$
Q(h)=-\int_{h}^{\infty} P(v) d v .
$$

This energy is monotone decreasing, with the rate of dissipation given by

$$
\frac{d \mathcal{E}}{d t}=-\int_{\Omega} f(h)|\nabla p|^{2} d A \leq 0
$$

In one dimension the local minimizers of the energy correspond to states with a constant uniform pressure, $p=\bar{p}$, and they solve the semilinear elliptic equation

$$
\bar{p}=P(h)-\Delta h \quad \text { on } \quad \Omega
$$

with Neumann boundary conditions. Solutions satisfy the compatibility condition

$$
\bar{p}=\frac{1}{\mathcal{A}} \int_{\Omega} P(h) d A .
$$

We consider a two-dimensional thin film resulting in a one-dimensional PDE for the film thickness $h . \Omega$ is given as an interval $(0, L)$ and $h$ solves the equation

$$
\frac{\partial h}{\partial t}+\frac{\partial}{\partial x}\left(f(h) \frac{\partial}{\partial x}\left[\frac{\partial^{2} h}{\partial x^{2}}-P(h)\right]\right)=0 .
$$

\subsection{Minimizers}

In this section we discuss the existence theory for the one dimensional problem and prove existence of a smooth global minimizer of the Liapunov functional (79). For a pressure of the form (76), we have the following theorem for positive initial data

Theorem 4.1 Consider initial data satisfying

$$
h_{0}>0, h_{0} \in H^{1}(\Omega), \text { and } \frac{1}{2} \int_{\Omega}\left|\partial_{x} h_{0}\right|^{2} d x+\int_{\Omega} Q\left(h_{0}\right) d x<\infty,
$$

and assume that $0<n<m, m \geq 3$ in (76). Then a unique positive smooth solution of (73-76) exists for all $t>0$. 
Proof Following arguments from previous papers [13, 22] it suffices to derive a priori pointwise upper and lower bounds for the solution. We derive an a priori bound on the $H^{1}$ norm and show this implies pointwise bounds. Then uniform parabolicity implies the solution is completely smooth. Similar arguments are presented in [13] for the case $P(h)=0$ and in [22] for a destabilizing non-singular $P(h)$.

First we note that the Liapunov functional implies that for any time $T>0$,

$$
\frac{1}{2} \int_{\Omega}\left|\partial_{x} h(T)\right|^{2} d x \leq \frac{1}{2} \int_{\Omega}\left|\partial_{x} h_{0}\right|^{2} d x+\int_{\Omega} Q\left(h_{0}\right) d x-\int_{\Omega} Q(h(T)) d x .
$$

The initial data is in $H^{1}$ and positive. Since $m>n,-Q(h)$ has an a priori upper bound independent of $h$, implying an a priori bound for $\int\left|\partial_{x} h\right|^{2} d x$ at the later time $T$. In one space dimension this immediately implies an a priori pointwise upper bound on $h$ and also on the $C^{1 / 2}$ norm, at time $T$.

To show that $h$ has a pointwise a priori lower bound at time $T$ note that the above implies $\int Q(h(T)) d x \leq C$. Let $h_{\min }=\min _{\Omega} h$ be attained at $x_{0}$. By Hölder continuity, $h(x) \leq u_{\min }+C_{H}\left|x-x_{0}\right|^{1 / 2}$ where $C_{H}$ is the Hölder constant. Hence:

$$
C>\int Q(h) d x \geq C(\epsilon) \int\left(h_{\min }+C_{H}\left|x-x_{0}\right|^{1 / 2}\right)^{-m+1} d x+O(1) \geq C(\epsilon) \eta\left(h_{\min }\right)+O(1)
$$

where $\eta\left(h_{\min }\right)=-\log h_{\min }$ for $m=3$ and $\eta\left(h_{\min }\right)=h_{\min }^{3-m}$ for $m>3$. Thus the solution is forbidden to go below a positive threshold for $m \geq 3$. Note that all the bounds depend strongly on $\epsilon$ as it nears zero. In particular these results are not true for the case $\epsilon=0$.

The long time behavior of solutions of the PDE is related to minimizers of the Liapunov functional (79). Define $Q(h)$ to be $\infty$ for $h \leq 0$; we can then prove the following results concerning the global minimizer.

Theorem 4.2 [28] Let $\Omega$ be a bounded domain of class $C^{0,1}$, let $\bar{h}>0$ and assume $0<n<m$ in (76). Then a global minimizer of $\mathcal{E}(\cdot)$ (79) exists in the class $V:=\left\{h \in H^{1}(\Omega): \int_{\Omega} h d x=\right.$ $\bar{h}\}$. In particular, $h>0$ almost everywhere in $\Omega$.

One can go on to prove that the global minimizer satisfies the same positivity result as in Theorem 4.2. Moreover the minimizer satisfies the steady state ODE:

Theorem 4.3 [28] Let $m \geq 3$ and $0<n<m$ in (76). Then a minimizer $h$ as constructed in theorem 4.2 is a solution of the $O D E$ on $\Omega=\{x \mid 0 \leq x \leq L\}$,

$$
\begin{gathered}
P(h)-h_{x x}=\bar{p} \\
h_{x}(0)=h_{x}(L)=0, \quad \frac{1}{L} \int_{0}^{L} h d x=\bar{h} .
\end{gathered}
$$

Moreover, $h$ is smooth. 


\subsection{One-dimensional steady-state solutions}

From Theorem 3 we know that all minimizers of $\mathcal{E}$ are smooth steady state solutions on $0 \leq x \leq L$ satisfying

$$
\begin{gathered}
P(h)-h_{x x}=\bar{p}, \\
h_{x}(0)=h_{x}(L)=0, \quad \bar{p}=\frac{1}{L} \int_{0}^{L} P(h) d x .
\end{gathered}
$$

In this section we examine the number and structure of steady state solutions.

While aspects of this problem parallel the Cahn-Hilliard and constrained Allen-Cahn equations $[5,6,40,53,75]$, the nonlinear function $P(h)$ in $(86)$ has a different form than considered in most previous works and leads to a different structure of the bifurcation diagram.

Equation (86) can be written as an autonomous phase plane system,

$$
h_{x}=v, \quad v_{x}=P(h)-\bar{p},
$$

where $\bar{p}$ is a control parameter. Observe that every Neumann solution of (86-87) on $x \in(0, L)$ can be extended by reflection symmetry to yield a periodic solution of $(88)$ on $x \in(0,2 L)$. Hence, a necessary condition for the existence of Neumann solutions is that (88) admits periodic solutions. Moreover the symmetry of (88) guarantees that periodic solutions on $[0,2 L]$ can be shifted to yield Neumann solutions on $[0, L]$.

Let $P_{\max }$ be the maximum of $P(h)$ on $h>0$. Observe that for any $P(h)$ of the form $(76)$ and $\bar{p} \in\left(0, P_{\max }\right),(88)$ has a unique elliptic center point at $h_{c}$ satisfying $P\left(h_{c}\right)-\bar{p}=0$ with $P^{\prime}\left(h_{c}\right)<0$. Note that for $\bar{p}<0$ there are no elliptic equilibria hence no periodic orbits and no Neumann solutions of (86). In addition, for $0 \leq \bar{p}<P_{\max }$, (88) has a hyperbolic saddle point at $h_{s} \sim \epsilon+\epsilon^{n+1} \bar{p} /(m-n)$ as $\epsilon \rightarrow 0$ with $h_{c}>h_{s}$. The stable and unstable manifolds of the saddle point form a homoclinic orbit that encloses $h_{c}$ and all of the periodic solutions of (88). Periodic solutions surrounding the center $h_{c}$ have a minimum greater than the saddle point value $h_{s}$. Therefore every non-trivial equilibrium solution is bounded from below by $h_{s}$, and thus $h(x)>\epsilon$.

Equation (87) has the trivial uniform constant solution $h=\bar{h}$ for any positive value $\bar{h}>0$; the corresponding average pressure is $\bar{p}=P(\bar{h})$. Branches of non-trivial solutions can bifurcate from the constant solutions only at singular points $\bar{h}_{*}$ where the linear operator $\mathcal{L}=P^{\prime}\left(\bar{h}_{*}\right)-\partial_{x x}$ has a non-trivial nullspace [65]; hence the bifurcation points are given by

$$
P^{\prime}\left(\bar{h}_{k}\right)=-k^{2} \pi^{2} / L^{2}, \quad k=1,2, \ldots, K .
$$

For $\epsilon>0, P^{\prime}(h)$ is bounded from below for $h>0$, therefore there are only a finite number of branches, $k=1,2, \ldots, K$. For each $k$, there are two roots, $\bar{h}_{k}^{+}>\bar{h}_{k}^{-}$, with $P^{\prime \prime}\left(\bar{h}_{k}^{+}\right)>0$ and $P^{\prime \prime}\left(\bar{h}_{k}^{-}\right)<0$. It is straightforward to show that as $\epsilon \rightarrow 0, K=O\left(\epsilon^{-(n+1) / 2}\right)$ by noting that the minimum scales like $P^{\prime}\left(h^{*}\right)=O\left(\epsilon^{-(n+1)}\right)$, where the critical point scales as $h^{*}=O(\epsilon)$. For $\epsilon=0$ there are a infinite number of upper solutions $\bar{h}_{k}^{+}$while the lower solutions $\bar{h}_{k}^{-}$ disappear.

We consider an interval of fixed length $L$ and examine the bifurcating branches of solutions as the total mass, $L \bar{h}=\int_{0}^{L} h(x) d x$, varies. For $\epsilon>0$, for each $k$ there is a branch of nontrivial steady state solutions that bifurcates from $\bar{h}_{k}^{+}$and reconnects at $\bar{h}_{k}^{-}$. This can be proved rigorously for $m$ and $n$ satisfying the following conditions. 


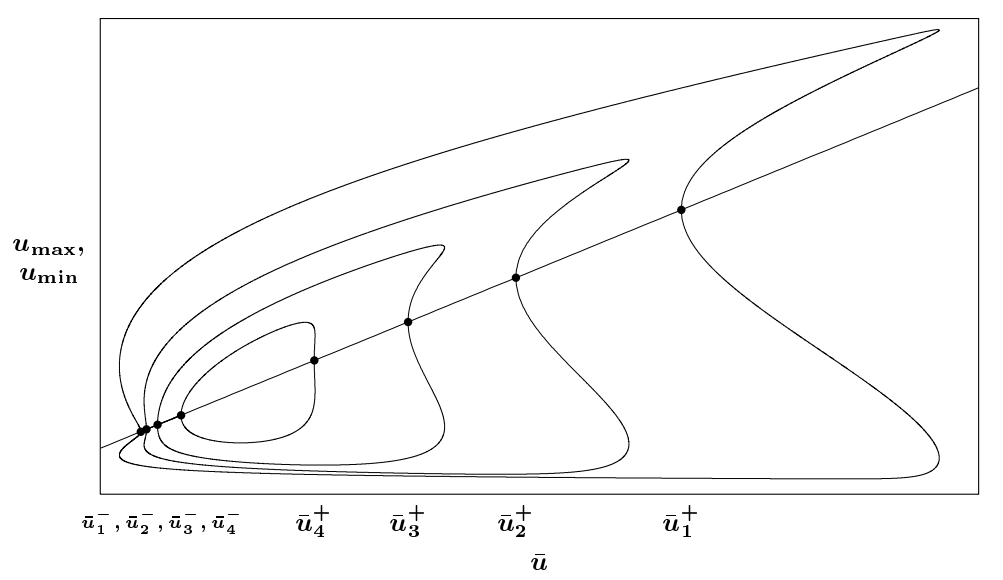

Figure 9: Bifurcation diagram of equilibria for $(76)$ with $(n, m)=(3,4)$ and $\epsilon=0.14$, yielding $K=4$ branches.

Definition 4.4 We call a pair of positive exponents $(n, m)$ in (76) admissible if they satisfy $m>n$ and either (I) or (II):

$$
\begin{aligned}
& \left\{n>1 / 2 \text { and } 3 n^{2}-10 m n+3 m^{2}-m-n+2>0\right\} \\
& \left\{3 n^{2}-14 m n+3 m^{2}-2 m-2 n+7<0\right\} .
\end{aligned}
$$

The following theorem is proved in [28].

Theorem 4.5 Consider $P(h)$ as given in (76) and admissible $(n, m)$ in the sense of Definition 4.4. Then, for each positive integer $k \leq K$ there exists a non-trivial branch of solutions to (86), (87). Parametrized by $\bar{p} \in\left(P\left(\bar{h}_{k}^{+}\right), P\left(\bar{h}_{k}^{-}\right)\right)$, each branch of non-trivial solutions bifurcates from $\bar{h}_{k}^{+}$and reconnects to the constant state branch at $\bar{h}_{k}^{-}$. Furthermore, all solutions of $(86,87)$ are either trivial constant solutions or lie on one of these bifurcating branches.

Figure 9 shows the bifurcation diagram for the set of equilibria of $(86,87)$. At each $\bar{h}$, the diagram gives the maximum and minimum values of each solution $h(x)$. Specifying two out of three of the values $h_{\min }, h_{\max }, \bar{h}$, specifies an equilibrium solution $h(x)$, unique up to the reflection symmetry, $x \rightarrow L-x$.

The local structure of the bifurcation diagram can computed analytically using perturbation methods. This results in an amplitude equation that predict either subcritical or supercritical pitchfork bifurcations at each of the points on the constant solution curve where an eigenvalue changes sign. For details, the reader is referred to [28].

\subsection{Stability of the equilibrium solutions}

In this section we consider the stability of equilibria with respect to mass-preserving perturbations. We begin with the linear analysis for uniform constant equilibrium solutions and go on to make more global statements for the entire set of equilibrium solutions.

If the initial data for the PDE $(84)$, with $f(h), P(h)$ given by $(75,76)$ is near an equilibrium $\underline{h}(x)$, then it is convenient to write the solution in the form $h(x, t)=\underline{h}(x)+\tilde{h}(x, t)$, where $\tilde{h}$ 
is a zero-mean perturbation. Linearizing (84) yields the evolution equation for $\tilde{h}$,

$$
\frac{\partial \tilde{h}}{\partial t}=\mathcal{L} \tilde{h} \equiv \partial_{x}\left(f(\underline{h}) \partial_{x}\left[P^{\prime}(\underline{h})-\partial_{x x}\right]\right) \tilde{h} .
$$

with the corresponding linearized Neumann boundary conditions (77),

$$
\partial_{x x x} \tilde{h}=0, \quad \partial_{x} \tilde{h}=0, \quad \text { at } x=0,1 .
$$

The choice $\tilde{h}(x, t)=\hat{h}(x) e^{\lambda t}$ in (90) yields the associated Neumann eigenvalue problem, $\mathcal{L} \hat{h}=\lambda \hat{h}$.

We note that the linear stability of the uniform constant solutions $\underline{h}(x)=\bar{h}$ can be computed directly [97]. For these states

$$
\lambda_{\ell}=-f(\bar{h}) \ell^{2} \pi^{2}\left(P^{\prime}(\bar{h})-\ell^{2} \pi^{2}\right), \quad \ell=1,2,3 \cdots
$$

with corresponding eigenmodes $A \cos (\pi \ell x)$.

The operator (90) is the composition of two second-order operators, $\mathcal{L} \hat{h}=\overline{\mathcal{M}} \overline{\mathcal{L}} \hat{h}$, where the mobility operator, $\overline{\mathcal{M}}$, and nonlocal operator, $\overline{\mathcal{L}}$, are respectively

$$
\overline{\mathcal{M}} v \equiv \partial_{x}\left(f(\underline{h}) \partial_{x} v\right), \quad \overline{\mathcal{L}} w \equiv P^{\prime}(\underline{h}) w-w_{x x}-\frac{1}{L} \int_{0}^{L} P^{\prime}(\underline{h}) w d x
$$

for zero-mean $w(x)$ satisfying Neumann boundary conditions. We note that $\mathcal{L}$ is self-adjoint with respect to a weighted $H^{-1}$ norm using an inner product defined by the mobility. For every zero-mean function $w(x)$, there exists a unique zero-mean potential, $W$, such that $\overline{\mathcal{M}} W=w$. The weighted $H^{-1}$ inner-product of two zero-mean functions $w$ and $v$ is then $\langle w, v\rangle \equiv \int f(\underline{h}) \partial_{x} W \partial_{x} V d x$. This norm can be used to describe the thin film evolution as a gradient flow in $H^{-1}$. As the operator $\mathcal{L}$ is self-adjoint and the domain is compact, the spectrum is real and discrete $[45,69]$. We note that related results on the properties of the linearized spectrum can be obtained from analysis of a Rayleigh quotient, as considered by Bates and Fife for the Cahn-Hilliard equation [9] and by Laugesen and Pugh [68, 69] for equations of the type (5).

In the case of constant mobility $f(h)=c$, Bai et al. [5] and Grinfeld and Novick-Cohen [53] showed that the properties of the spectrum of (90) can be obtained from analysis of the second order problem for $\overline{\mathcal{L}}$. Since the equilibria are smooth and bounded away from zero, the problem with non-constant mobility $f(u)$ can be treated equivalently; the operator $\overline{\mathcal{M}}$ is invertible and hence $\mathcal{L} \hat{h}=\lambda \hat{h}$ can be written as $\overline{\mathcal{L}} \hat{h}=\lambda \overline{\mathcal{M}}^{-1} \hat{h}$. These results directly extend to (90) using the homotopy

$$
\overline{\mathcal{L}} \hat{h}=\lambda\left[\alpha+(1-\alpha) \overline{\mathcal{M}}^{-1}\right] \hat{h},
$$

where $\alpha=0$ gives the fourth order operator $\mathcal{L}$ and $\alpha=1$ gives the second order operator $\overline{\mathcal{L}}$. Lemma 1.2 in [53] and Theorem 3.1 in [5] show that the number of positive and negative eigenvalues of (94) is independent of $0 \leq \alpha \leq 1$. Furthermore, Grinfeld and Novick-Cohen's Theorem 5.1 [53] for excluding $\lambda=0$ from the spectrum of $\mathcal{L}$ directly extends to our problem and can be stated as 
Theorem 4.6 Consider the linearized equation (90) with $f(h), P(h)$ given by (75, 76). Then, away from the bifurcation points $\bar{h}_{k}^{ \pm}$on the line of constant solutions, and apart from turning points, $\lambda=0$ is not in the spectrum of (90) on the branches of non-trivial solutions. No secondary bifurcations are possible.

For the Neumann problem for (5), Laugesen and Pugh have proved that equilibrium solutions with interior critical points are unstable (see Theorem 4 [69]). We now combine these results to prove the existence of a family of stable non-trivial solutions for (84).

Theorem 4.7 For problem (84) with $P(h)$ in (76) satisfying Theorem 4.5 and $f(h)$ given by (75), there exists an interval $I=\left(\bar{h}_{1}^{\min }, \bar{h}_{1}^{\max }\right)$ containing $\left(\bar{h}_{1}^{-}, \bar{h}_{1}^{+}\right)$such that for each $\bar{h} \in I$, there exists a stable non-trivial steady state solution. These stable monotone solutions lie on the first branch of solutions, $k=1$.

Figure 9 with $\epsilon=0.14$ shows a $k=1$ branch with a very simple structure. There is precisely one turning point for each subcritical bifurcation. We conjecture that this is generically true for this problem with $P(h)$ of the form (76). In this case, we can determine the number of positive eigenvalues for all solutions along the first bifurcating branch. The first branch has two unstable portions, one near each of the bifurcation points. By Theorem 4.7, on the portion of the non-trivial branch between the turning points, all of the solutions must be stable. In the next section we study the asymptotic structure of this branch in the limit as $\epsilon \rightarrow 0$.

Corollary 4.8 For $k=2, \ldots, K$, a subset of solutions along the $k^{\text {th }}$ branch of non-trivial solutions (connecting $h_{k}^{-}$to $h_{k}^{+}$) is stable with respect to perturbations with the same $k$-fold symmetry.

This result follows directly from Theorem 4.7 by noting that $k^{\text {th }}$ bifurcating branch for the problem with domain size $L$ is the first bifurcating branch for the problem with domain size $L / k$. Instability of these reduced-domain-stable solutions to perturbations on the full domain is called coarsening [9].

\subsection{Asymptotic behavior of equilibrium solutions}

We now study the structure of the equilibrium solutions of $(86,87)$ in the limit that $\epsilon \rightarrow 0$. This branch has subcritical pitchfork bifurcations at $\bar{h}_{1}^{+}$and $\bar{h}_{1}^{-}$. Turning points occur on this branch at $\bar{h}_{1}^{\max }$ and $\bar{h}_{1}^{\text {min }}$, respectively describing the maximum and minimum average film thicknesses for non-trivial solutions. As described above, the turning points separate families of stable and unstable solutions on this branch. The unstable solutions have a finite amplitude structure, with $h_{\min }=O(1)$ as $\epsilon \rightarrow 0$, while the stable solutions are localized droplets connected by an ultra-thin film with thickness $h \sim \epsilon$ (see Figure 10) and approach the homoclinic solution of (88). These stable droplet solutions concentrate mass as a $\delta$ distribution in the limit of vanishing $\epsilon$. Moreover, using matched asymptotic analysis [28] one can obtain an estimate for the turning point $\bar{h}_{1}^{\max }$ as a function of $\epsilon$.

As $\epsilon \rightarrow 0$, for each allowable fixed value of $\bar{h} \in\left(\bar{h}_{1}^{\min }, \bar{h}_{1}^{\max }\right)$, we get the solution

$$
h(x) \sim \begin{cases}\frac{3 \bar{h}}{2 \bar{x}^{3}}\left(\bar{x}^{2}-x^{2}\right) & 0 \leq x<\bar{x}=\left(\frac{9}{2} \bar{h}^{2} /|Q(\epsilon)|\right)^{1 / 4}, \\ \epsilon & \bar{x}<x \leq 1 .\end{cases}
$$




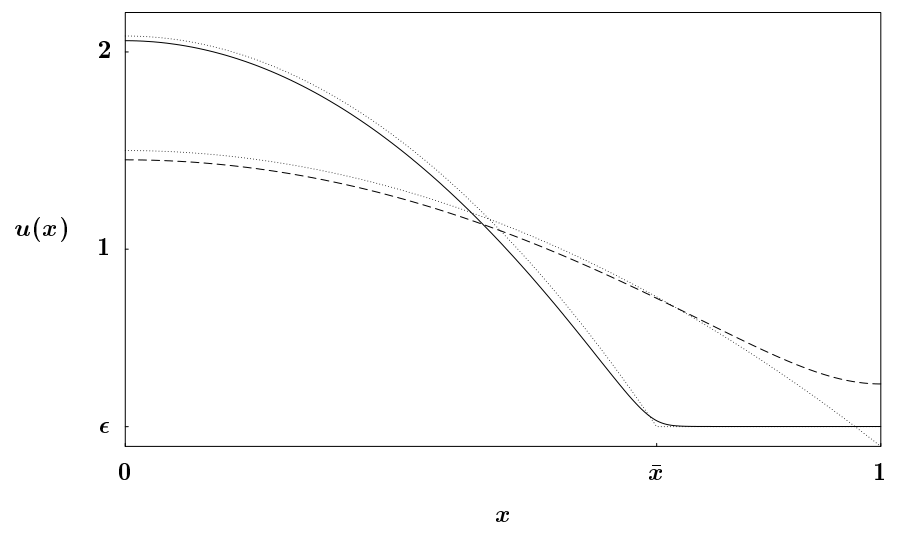

Figure 10: The stable (solid) and unstable (dashed) equilibrium solutions, and the leadingorder asymptotic approximations (dotted), for $\epsilon=0.1$ and $(n, m)=(3,4)$ with $\bar{h}=1$, and the leading-order asymptotic approximations (dotted).

From the form of $Q$, for $\epsilon \rightarrow 0, Q(\epsilon)=O\left(\epsilon^{-(n-1)}\right)$ and we obtain

$$
\bar{x} \sim \sqrt{\bar{h}} O\left(\epsilon^{(n-1) / 4}\right) \rightarrow 0, \quad \bar{p} \sim O\left(\epsilon^{-3(n-1) / 4}\right) / \sqrt{\bar{h}} \rightarrow \infty .
$$

Hence for $\epsilon \rightarrow 0,(95)$ is a delta sequence, which converges to a Dirac $\delta$-distribution with mass $\bar{h}, \bar{h} \delta(x)$. As $\epsilon$ decreases, (95) approaches a parabolic profile with fixed area and support that vanishes as $\epsilon \rightarrow 0$. An estimate of the maximum mass $\bar{h}_{1}^{\max }$ of the first branch of the bifurcation diagram is given by the intersection of the two asymptotic families of solutions. This occurs when $h_{\max } \sim 3 \bar{h} / 2$ and (96) are set equal to yield

$$
\bar{h} \leq \bar{h}_{1}^{\max } \sim \frac{2}{9} \sqrt{2|Q(\epsilon)|} .
$$

\subsection{Discussion}

In our study, for finite $\epsilon>0$, the conjoining pressure term in (76) regularizes the $\epsilon=0$ finitetime rupture problem. For $\epsilon>0$ the evolution is well-posed through topological transitions, as in diffusive interface models for Hele-Shaw flows. And, for $\epsilon \rightarrow 0$, we will present numerical simulations of the dynamics of (84) that show the occurrence of concentrations.

To examine the formation of mass concentrations as $\epsilon \rightarrow 0$ after rupture, we consider a sequence of numerical solutions $h^{\epsilon}(x, t)$ of the initial value problem for (84) with $L=1$, $f(h)=h^{3}$, and $P(h)=h^{-3}-\epsilon h^{-4}$, with the monotone decreasing initial data $h_{0}(x)=$ $0.4+0.002 \cos (\pi x)$. Figure 11 shows the maximum and minimum of the solution as a function of time for the following values of $\epsilon$ : $0.1,0.05,0.025,0.0125,0.00625$, and $\epsilon=0$. The $\epsilon=0$ problem exhibits finite-time rupture at time $T_{R} \approx 0.041$. For $\epsilon>0$, the solutions do not rupture, but each "de-wets" shortly after $T_{R}$, that is the minimum of each solutions becomes small, $h_{\min }(t)=O(\epsilon)$. Thereafter, the $\epsilon>0$ solutions rapidly converge to the unique non-trivial stable steady state solution for this problem. The graphs of the minimum and maximum of the solutions $h^{\epsilon}(x, t)$ shown in Figure 11 give one indicator of the convergence to the steady state. We find that for smaller values of $\epsilon$ the convergence to steady state happens on shorter time scales. Since the steady states converge to a Dirac $\delta$-distribution, 


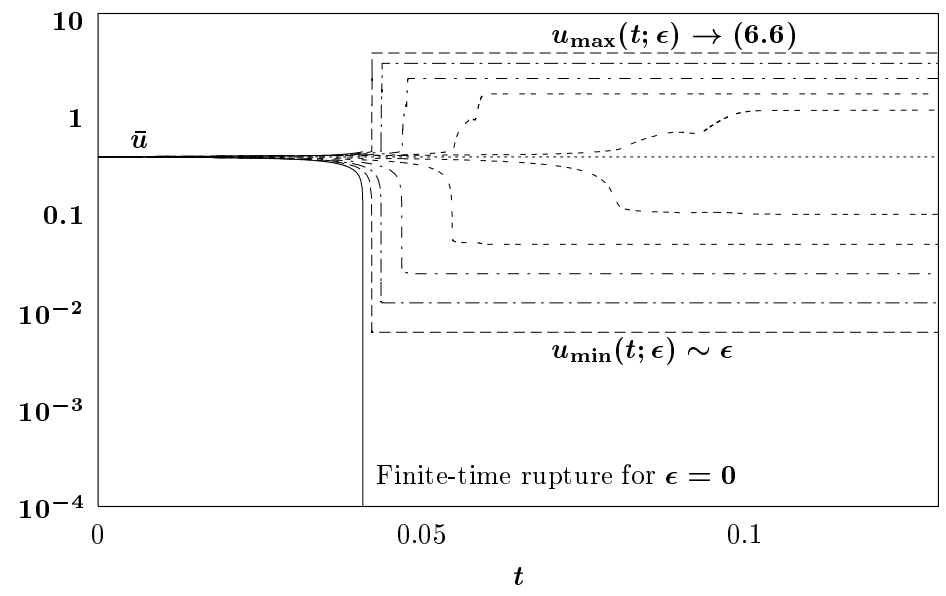

Figure 11: A sequence of dynamic simulations in the $\epsilon \rightarrow 0$ limit. Numerical results indicate convergence to the equilibrium rapidly after rupture for $\epsilon \rightarrow 0$.

the computations suggest that for each time after the rupture time $T_{R}$, the time-dependent solution sequence converges to a $\delta$-distribution, i.e. for all $t \geq 0$,

$$
\lim _{\epsilon \rightarrow 0} h^{\epsilon}(x, t)= \begin{cases}h^{0}(x, t), & t \leq T_{R}, \\ \bar{h} \delta(x), & t>T_{R} .\end{cases}
$$

Thus concentrations occur in this solution sequence for $\epsilon \rightarrow 0$. In fact the only estimate, independent of $\epsilon$, that is known for the PDE is the $L^{1}$ norm, that is the conservation of mass. This simulation of $h^{\epsilon}(x, t)$ suggests that sharper analytical control is not possible. Finally it is clear from this simulation that there is a droplet formation timescale, $T_{D}$, i.e. the time it takes for the solution to converge to a parabolic droplet after the onset of dewetting at $T_{R}$. This preliminary numerical study suggests that $T_{D} \rightarrow 0$ as $\epsilon \rightarrow 0$.

To briefly study the influence of the limit $\epsilon \rightarrow 0$ on the dynamics of topological transitions (described in [78] as "morphological phase separation"), we look at simulations illustrating long-time coarsening dynamics in (84). We use the algorithm in [55] to compute the solution of (84) with $f(h)=h^{3}, P(h)=h^{-3}-\epsilon h^{-4}, L=20$, and the initial data $h_{0}(x)=1+$ $0.005 \sin \left(6(x-13)^{2}\right)$, for $\epsilon=0.1$ and $\epsilon=0.01$. Unlike the simple monotone initial data used in Figure 11, this new initial data has the potential to form complicated patterns of droplets that may depend sensitively on the structure of $h_{0}(x)$. As shown in Figure 12, these simulations illustrate the dynamics of dewetting (or formation of "dry spots") and coarsening starting from small perturbations of a flat thin film. Similar results have been observed in other studies for fixed values of $\epsilon[73,76,78]$; our interest here is the dependence of the dynamics on $\epsilon$, in the limit that $\epsilon \rightarrow 0$. Figure 12 shows the results for $\epsilon=0.1$ and $\epsilon=0.01$. In both situations, after an initial period of dewetting dynamics, the solutions converge to a series of droplets, at arbitrary positions in the domain, generally with different masses, all connected by an ultra-thin film. Each of these droplets is close to an equilibrium solution, and in fact they persist for very long times. However, from Section 4.3, we know that there can not be any stable equilibria with interior extrema [69], hence these states are unstable. These states are called meta-stable, and their long-time dynamics has been studied 


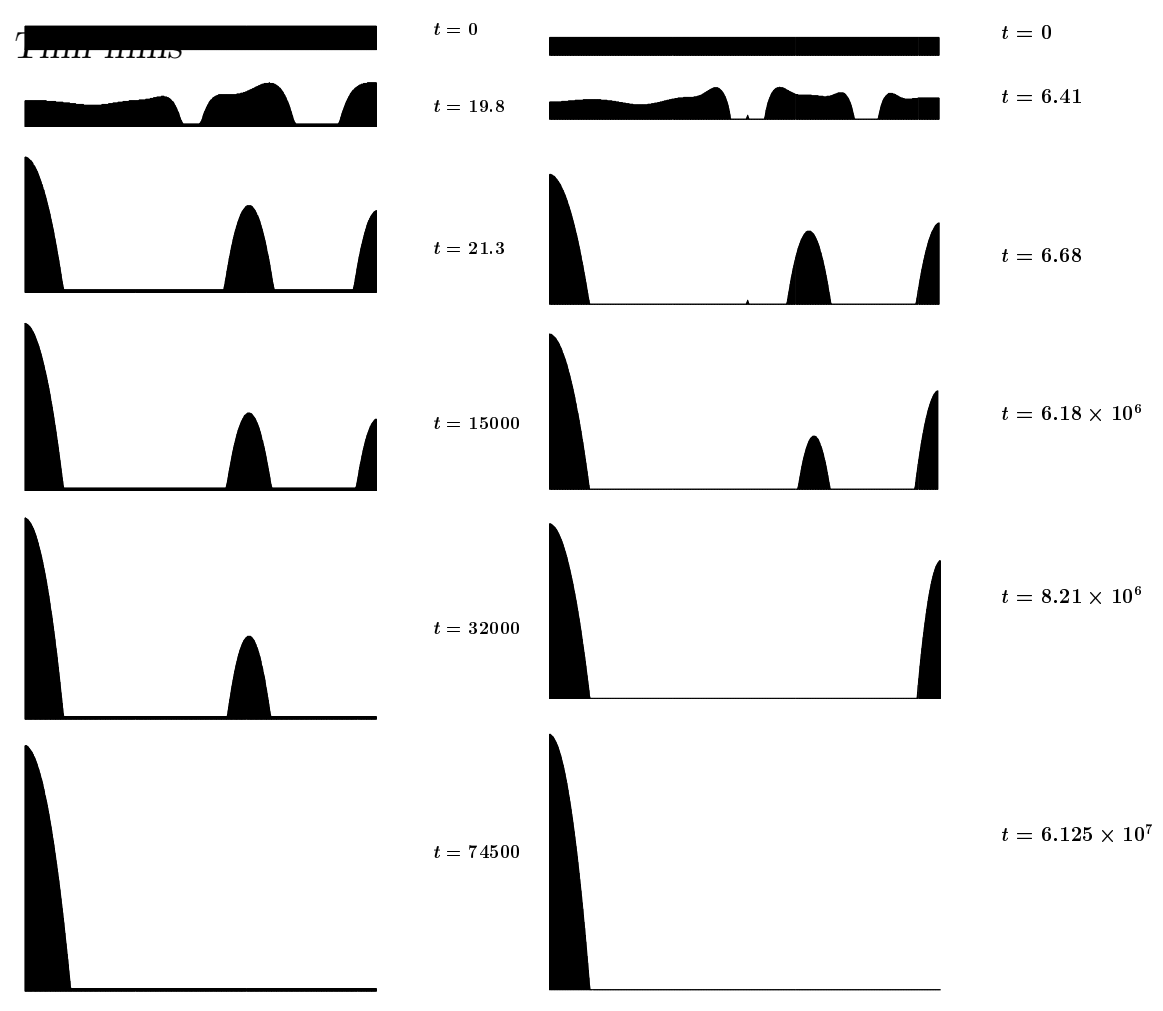

Figure 12: The long time dynamics for (84) with $n=3, m=4$ for $\epsilon=0.1$ (left) and $\epsilon=0.01$ (right). Note that the time-scale for the coarsening process increases as $\epsilon \rightarrow 0$ and that the final state converges to the global minimizer.

for Cahn-Hilliard equations [82, 95]. The long-time dynamics are characterized by coarsening instabilities that ultimately lead to a final stable droplet which is the global minimizer. The time needed for the coarsening process depends on $\epsilon$, with smaller $\epsilon$, requiring more time to evolve to the global minimizer. Thus there is a coarsening time scale, $T_{C}$, that describes the time it takes for the number of droplets in the solution to decrease. As suggested by Figure 12, this time scale increases, $T_{C} \rightarrow \infty$, as $\epsilon \rightarrow 0$, a dramatically different dependence on $\epsilon$ than the droplet formation time-scale, $T_{D} \rightarrow 0$. A model for the coarsening dynamics, in terms of droplet motion and mass transfer, is developed in [49].

\section{Acknowledgements}

This work is supported by NSF grant DMS-0074049 and ONR grant N000140110290.

\section{References}

[1] R. Abeyaratne and J.K. Knowles. Implications of viscosity and strain gradient effects for the kinetics of propagating phase boundaries in solids. SIAM J. Appl. Math., 51:1205$1221,1991$.

[2] D. J. Acheson. Elementary Fluid Dynamics. Oxford Univ. Press, Oxford, UK, 1990. 
[3] Robert Almgren, Andrea L. Bertozzi, and Michael P. Brenner. Stable and unstable singularities in the unforced Hele-Shaw cell. Phys. Fl., 8(6):1356-1370, June 1996.

[4] D. G. Aronson and J. Graveleau. A self-similiar solution to the focusing problem for the porous medium equation. European J. Appl. Math., 4, 1993.

[5] F. S. Bai, C. M. Elliott, A. Gardiner, A. Spence, and A. M. Stuart. The viscous Cahn-Hilliard equation. I. Computations. Nonlinearity, 8(2):131-160, 1995.

[6] F. S. Bai, A. Spence, and A. M. Stuart. Numerical computations of coarsening in the one-dimensional Cahn-Hilliard model of phase separation. Phys. D, 78(3-4):155-165, 1994.

[7] G. I. Barenblatt. Scaling, Self-similarity, and Intermediate Asymptotics. Cambridge University Press, New York, 1996.

[8] John W. Barrett, James F. Blowey, and Harald Garcke. Finite element approximation of a fourth order degenerate parabolic equation. Numer. Math., 80(4):525-556, October 1998.

[9] P. W. Bates and P. C. Fife. Spectral comparison principles for the Cahn-Hilliard and phase-field equations, and time scales for coarsening. Phys. D, 43(2-3):335-348, 1990.

[10] R. M. Beam and R. F. Warming. Alternating direction implicit methods for parabolic equations with a mixed derivative. SIAM J. Sci. Stat. Comput., 1:131-159, 1980.

[11] Elena Beretta, Michiel Berstch, and Roberta Dal Passo. Nonnegative solutions of a fourth order nonlinear degenerate parabolic equation. Arch. Rational Mech. Anal., 129:175-200, 1995.

[12] F. Bernis. Change in sign of the solutions to some parabolic problems. In V. Lakshmikantham, editor, Nonlinear analysis and applications (Arlington, Tex., 1986), volume 109, pages 75-82. Marcel Dekker, New York, 1987.

[13] F. Bernis and A. Friedman. Higher order nonlinear degenerate parabolic equations. $J$. Diff. Equations, 83:179-206, 1990.

[14] F. Bernis, L. A. Peletier, and S. M. Williams. Source type solutions of a fourth order nonlinear degenerate parabolic equation. Nonlinear Analysis, Theory, Methods and Applications, 18(3):217-234, 1992.

[15] Francisco Bernis. Finite speed of propagation and continuity of the interface for slow viscous flows. Adv. Differential Equations, 1(3):337-368, 1996.

[16] Francisco Bernis. Finite speed of propagation for thin viscous flows when $2 \leq n<3$. C. R. Acad. Sci. Paris, 322:1169-1174, 1996.

[17] A. Bertozzi, A. Münch, M. Shearer, and K. Zumbrun. Stability of compressive and undercompressive thin film travelling waves. European J. of Appl. Math., 12(3):253291, 2001. 
[18] A. L. Bertozzi. Loss and gain of regularity in a lubrication equation for thin viscous films. In J. I. Díaz, M. A. Herrero, A. Liñán, and J. L. Vázquez, editors, Free Boundary Problems: Theory and Applications, volume 323 of Pitman Research Notes in Mathematics Series, pages 72-85. Longman House, 1995. Proceedings of the International Colloquium on Free Boundary Problems, Toledo, Spain, June 1993.

[19] A. L. Bertozzi and M. P. Brenner. Linear stability and transient growth in driven contact lines. Phys. Fluids, 9(3):530-539, March 1997.

[20] A. L. Bertozzi, A. Münch, X. Fanton, and A. M. Cazabat. Contact line stability and 'undercompressive shocks' in driven thin film flow. Phys. Rev. Lett., 81(23):5169-5172, December 1998.

[21] A. L. Bertozzi, A. Münch, and M. Shearer. Undercompressive shocks in thin film flows. Physica D, 134(4):431-464, 1999.

[22] A. L. Bertozzi and M. C. Pugh. Long-wave instabilities and saturation in thin film equations. Comm. Pure Appl. Math., LI:625-661, 1998.

[23] A. L. Bertozzi and M. Shearer. Existence of undercompressive travelling waves in thin film equations. SIAM J. Math. Anal., 32(1):194-213, 2000.

[24] A.L. Bertozzi and M. Pugh. The lubrication approximation for thin viscous films: regularity and long time behavior of weak solutions. Comm. Pur. Appl. Math., 49(2):85123, February 1996.

[25] Andrea L. Bertozzi. Symmetric singularity formation in lubrication-type equations for interface motion. SIAM J. Applied Math., 56(3):681-714, June 1996.

[26] Andrea L. Bertozzi. The mathematics of moving contact lines in thin liquid films. Notices of the American Math. Soc., 45(6):689-697, 1998.

[27] Andrea L. Bertozzi, Michael P. Brenner, Todd F. Dupont, and Leo P. Kadanoff. Singularities and similarities in interface flow. In L. Sirovich, editor, Trends and Perspectives in Applied Mathematics, volume 100 of Applied Mathematical Sciences, pages 155-208. Springer-Verlag, New York, 1994.

[28] Andrea L. Bertozzi, Günther Grün, and Thomas P. Witelski. Dewetting films: bifurcations and concentrations. Nonlinearity, 14(6):1569-1592, 2001.

[29] J. Bischof, D. Scherer, S. Herminghaus, and P. Leiderer. Dewetting modes of thin metallic films: Nucleation of holes and spinodal dewetting. Phys. Rev. Let., 77(8):15361539, 1996.

[30] Stephanella Boatto, Leo Kadanoff, and Piero Olla. Travelling wave solutions to thin film equations. Phys. Rev. E, 48:4423, 1993.

[31] Anne Bourlioux and Andrew J. Majda. Theoretical and numerical structure of unstable detonations. Phil. Trans. R. Soc. Lond. A., 350:29-69, 1995. 
[32] R. Buckingham, M. Shearer, and A. L. Bertozzi. Thin films traveling waves and the navier slip condition, 2002.

[33] J. P. Burelbach, S. G. Bankoff, and S. H. Davis. Nonlinear stability of evaporating/condensing liquid films. J. Fluid Mech., 195:463-494, 1988.

[34] Phillippe Carles and Anne-Marie Cazabat. The thickness of surface-tension-gradientdriven spreading films. J. Coll. Interfac. Sci., 157:196-201, 1993.

[35] A. M. Cazabat, F. Heslot, S. M. Troian, and P. Carles. Finger instability of this spreading films driven by temperature gradients. Nature, 346(6287):824-826, August 1990.

[36] Peter Constantin, Todd F. Dupont, Raymond E. Goldstein, Leo P. Kadanoff, Michael J. Shelley, and Su-Min Zhou. Droplet breakup in a model of the Hele-Shaw cell. Physical Review E, 47(6):4169-4181, June 1993.

[37] P.G. de Gennes. Wetting: Statics and dynamics. Rev. Mod. Phys., 57:827, 1985.

[38] Todd F. Dupont, Raymond E. Goldstein, Leo P. Kadanoff, and Su-Min Zhou. Finitetime singularity formation in Hele Shaw systems. Physical Review E, 47(6):4182-4196, June 1993.

[39] E. B. Dussan V and S. Davis. On the motion of a fluid-fluid interface along a solid surface. J. Fluid Mech., 65:71-95, 1974.

[40] J. C. Eilbeck, J. E. Furter, and M. Grinfeld. On a stationary state characterization of transition from spinodal decomposition to nucleation behaviour in the Cahn-Hilliard model of phase separation. Phys. Lett. A, 135(4-5):272-275, 1989.

[41] M. Elbaum, S. G. Lipsom, and J. F. Wettlaufer. Evavoration preempts complete wetting. Europhysics Letters, 29(6):457-462, 21995.

[42] M. H. Eres, L. W. Schwartz, and R. V. Roy. Fingering phenomena for driven coating films. Phys. Fl., 12(6):1278-1295, 2000.

[43] T. Erneux and D.Gallez. Can repulsive forces lead to stable patterns in thin liquid films? Phys. Fluids, 9(4):1194-1196, 1997.

[44] L. C. Evans. Weak convergence methods for nonlinear partial differential equations. Number 74 in CBMS Lecture Notes. American Mathematical Society, 1990.

[45] Lawrence C. Evans. Partial Differential Equations, volume 3A and B of Berkeley Mathematics Lecture Notes. Center for Pure and Applied Mathematics, Univ. of California, Berkeley, 1993.

[46] X. Fanton, A. M. Cazabat, and D. Quéré. Thickness and shape of films driven by a Marangoni flow. Langmuir, 12(24):5875-5880, 1996.

[47] Raul Ferreira and Francisco Bernis. Source-type solutions to thin-film equations in higher dimensions. Eur. J. of Appl. Math., 8(5):507-524, 1997. 
[48] Nathalie Fraysse and George M. Homsy. An experimental study of rivulet instabilities in centrifugal spin coating of viscous Newtonian and non-Newtonian fluids. Phys. Fluids, 6(4):1491-1504, April 1994.

[49] K. Glasner and T. P. Witelski. Coarsening dynamics of dewetting films. preprint, 2001.

[50] Sydney Goldstein, editor. Modern developments in fluid dynamics, volume 2. Dover Publications, Inc., 1965.

[51] A. A. Golovin, B. Y. Rubinstein, and L. M. Pismen. Effect of van der waals interaction on the fingering instability of thermally driven thin wetting films. Langmuir, 17:39303936, 2001.

[52] H. P. Greenspan. On the motion of a small viscous droplet that wets a surface. J. Fluid Mech., 84:125-143, 1978.

[53] M. Grinfeld and A. Novick-Cohen. The viscous Cahn-Hilliard equation: Morse decomposition and structure of the global attractor. Trans. Amer. Math. Soc., 351(6):23752406, 1999.

[54] G. Grün. On the convergence of entropy consistent numerical schemes for lubricationtype equations in multiple space dimensions. submitted for publication, 2000.

[55] G. Grün. On the numerical simulation of wetting phenomena. In W. Hackbusch and S. Sauter, editors, Proceedings of the 15th GAMM-Seminar Kiel, Numerical methods of composite materials. Vieweg-Verlag, Braunschweig, to appear.

[56] G. Grün and M. Rumpf. Nonnegativity preserving convergent schemes for the thin film equation. Numer. Math., 87(1):113-152, 2000.

[57] Patrick J. Haley and Michael J. Miksis. The effect of the contact line on droplet spreading. J. Fluid Mech., 223:57-81, 1991.

[58] S. Herminghaus, K. Jacobs, K. Mecke, J. Bischof, A. Fery, M. Ibn-Elhaj, and S. Schlagowski. Spinodal dewetting in liquid crystal and liquid metal films. Science, 282(5390):916-919, 1998.

[59] Chun Huh and L. E. Scriven. Hydrodynamic model of steady movement of a solid /liquid/fluid contact line. J. Colloid Interface Sci., 35:85-101, 1971.

[60] H. Huppert. Flow and instability of a viscous current down a slope. Nature, 300:427429, 1982.

[61] E. Isaacson, D. Marchesin, and B. Plohr. Transitional waves for conservation laws. SIAM J. Math. Anal., 21:837-866, 1990.

[62] Jacob N. Israelachvili. Intermolecular and surface forces. Academic Press, New York, 1992. second edition.

[63] Dawn E. Kataoka and Sandra M. Troian. A theoretical study of instabilities at the advancing front of thermally driven coating films. J. Coll. Int. Sci., 192:350-362, 1997. 
[64] Dawn E. Kataoka and Sandra M. Troian. Stabilizing the advancing front of thermally driven climbing films. J. Coll. Int. Sci., 203:335-344, 1998.

[65] J. B. Keller and S. Antman, editors. Bifurcation theory and nonlinear eigenvalue problems. W. A. Benjamin, Inc., New York-Amsterdam, 1969.

[66] R. Konnur, K. Kargupta, and A. Sharma. Instability and morphology of thin liquid films on chemically heterogeneous substrates. Phys. Rev. Let., 84(5):931-934, 2000.

[67] N. Kopell and L. N. Howard. Bifurcations and trajectories joining critical points. Advances in mathematics, 18:306-358, 1975.

[68] R. S. Laugesen and M. C. Pugh. Energy levels of steady states for thin film type equations. 2000.

[69] R. S. Laugesen and M. C. Pugh. Linear stability of steady states for thin film and Cahn-Hilliard type equations. Arch. Rat. Mech. Anal., 154(1):3-51, 2000.

[70] Peter D. Lax. Hyperbolic systems of conservation laws and the mathematical theory of shock waves, volume 11 of CBMS-NSF Regional conference series in applied mathematics. SIAM, Philadelphia, PA, 1973.

[71] F. Melo, J. F. Joanny, and S. Fauve. Fingering intability of spinning drops. Phys. Rev. Lett., 63(18):1958-1961, October 1989.

[72] V. S. Mitlin. Dewetting of a solid surface: analogy with spinodal decomposition. $J$. Coll. Int. Sci., 156:491-497, 1993.

[73] V. S. Mitlin and N. V. Petviashvili. Nonlinear dynamics of dewetting: kinetically stable structures. Phys. Lett. A, 192:323-326, 1994.

[74] A. Münch. Shock transitions in Marangoni-gravity driven thin film flow. Nonlinearity, 13:731-746, 2000.

[75] A. Novick-Cohen and L. A. Peletier. Steady states of the one-dimensional Cahn-Hilliard equation. Proc. Roy. Soc. Edinburgh Sect. A, 123(6):1071-1098, 1993.

[76] A. Oron and S. G. Bankoff. Dewetting of a heated surface by an evaporating liquid film under conjoining/disjoing pressures. J. Coll. Int. Sci., 218:152-166, 1999.

[77] A. Oron and S. G. Bankoff. Dynamics of a condensing liquid film under conjoining/disjoining pressures. Phys. Fluids, 13(5):1107-1117, 2001.

[78] A. Oron, S. H. Davis, and S. G. Bankoff. Long-scale evolution of thin liquid films. Rev. Mod. Phys., 69(3):931-980, 1997.

[79] Alexander Oron, Stephen H. Davis, and S. George Bankoff. Long-scale evolution of thin liquid films. Rev. Mod. Phys., 69(3):931-980, July 1997.

[80] L. A. Peletier. The porous media equation. In H. Amman et. al., editor, Applications of nonlinear analysis in the physical sciences, pages 229-241. Pitman, New York, 1981. 
[81] G. Reiter. Dewetting of thin polymer films. Phys. Rev. Lett., 68(1):75-78, 1992.

[82] L. G. Reyna and M. J. Ward. Metastable internal layer dynamics for the viscous Cahn-Hilliard equation. Methods Appl. Anal., 2(3):285-306, 1995.

[83] M. Schneemilch and A. M. Cazabat. Shock separation in wetting films driven by thermal gradients. Langmuir, 16(25):9850-9856, 2000.

[84] M. Schneemilch and A. M. Cazabat. Wetting films in thermal gradients. Langmuir, 16(23):8796-8801, 2000.

[85] L. W. Schwartz, R. V. Roy, R. R. Eley, and S. Petrash. Dewetting patterns in a drying liquid film. J. Coll. Int. Sci., 234(2):363-374, February 2001.

[86] Leonard W. Schwartz. Viscous flows down an inclined plane: Instability and finger formation. Phys. Fluids A, 1(3):443-445, March 1989.

[87] A. Sharma and R. Khanna. Pattern formation in unstable thin liquid films. Phys. Rev. Let., 81(16):3463-3466, 1998.

[88] A. Sharma and R. Khanna. Pattern formation in unstable thin liquid films under the influence of antagonistic short- and long-range forces. J. Chem. Phys., 110(10):49294936, 1999.

[89] M. Shearer, D. G. Schaeffer, D. Marchesin, and P. Paes-Leme. Solution of the Riemann problem for a prototype $2 \times 2$ system of non-strictly hyperbolic conservation laws. Arch. Rat. Mech. Anal., 97:299-320, 1987.

[90] M. Slemrod. Admissibility criteria for propagating phase boundaries in a van der Waals fluid. Arch. Rat. Mech. Anal., 81:301-315, 1983.

[91] V. M. Starov. Spreading of droplets of nonvolatile liquids over a flat solid. J. Colloid Interface Sci. USSR, 45, 1983.

[92] S. M. Troian, E. Herbolzheimer, S. A. Safran, and J. F. Joanny. Fingering instabilities of driven spreading films. Europhys. Lett., 10(1):25-30, September 1989.

[93] M. P. Valignat, S. Villette, J. Li, R. Barberi, R. Bartolino, E. Dubois-Violette, and A. M. Cazabat. Wetting and anchoring of a nematic liquid crystal on a rough surface. Phys. Rev. Lett., 77(10):1994-1997, 1996.

[94] F. Vandenbrouck, M. P. Valignat, and A. M. Cazabat. Thin nematic films: metastability and spinodal dewetting. Phys. Rev. Lett., 82(13):2693-2696, 1999.

[95] M. J. Ward. Metastable patterns, layer collapses, and coarsening for a one-dimensional Ginzburg-Landau equation. Stud. Appl. Math., 91(1):51-93, 1994.

[96] Malcolm B. Williams and Stephen H. Davis. Nonlinear theory of film rupture. Journal of Colloid and Interface Science, 90(1), November 1982.

[97] T. P. Witelski and A. J. Bernoff. Dynamics of three-dimensional thin film rupture. submitted to Physica D, 2000. 
[98] T. P. Witelski and M. Bowen. Adi methods for high order parabolic equations, 2001. submitted.

[99] Thomas P. Witelski and Andrew J. Bernoff. Stability of self-similar solutions for van der Waals driven thin film rupture. Physics of Fluids (Letters), 11(9):2443-2445, 1999.

[100] C.C. Wu. New theory of MHD shock waves. In M. Shearer, editor, Viscous Profiles and Numerical Methods for Shock Waves. SIAM, 1991.

[101] R. Xie, A. Karim, J. F. Douglas, C. C. Han, and R. A. Weiss. Spinodal dewetting of thin polymer films. Phys. Rev. Let., 81(6):1251-1254, 1998.

[102] W. W. Zhang and J. R. Lister. Similarity solutions for van der Waals rupture of a thin film on a solid substrate. Phys. Fluids, 11(9):2454-2462, 1999.

[103] L. Zhornitskaya and A. L. Bertozzi. Positivity-preserving numerical schemes for lubrication-type equations. SIAM J. Numer. Anal., 37(2):523-555 (electronic), 2000. 